\title{
Magnetic Helicity Condensation and the Solar Cycle
}

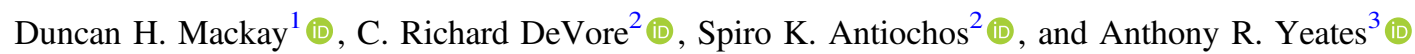 \\ ${ }^{1}$ School of Mathematics and Statistics, University of St. Andrews, North Haugh, St. Andrews, Fife, Scotland, KY16 9SS, UK; dhm@st-and.ac.uk \\ ${ }^{2}$ Heliophysics Science Division, NASA Goddard Space Flight Center, Greenbelt MD 20771, USA \\ ${ }^{3}$ Department of Mathematical Sciences, Durham University, Science Laboratories, South Road, Durham, DH1 3LE, UK \\ Received 2018 April 19; revised 2018 October 25; accepted 2018 October 25; published 2018 December 12
}

\begin{abstract}
Solar filaments exhibit a global chirality pattern where dextral/sinistral filaments, corresponding to negative/ positive magnetic helicity, are dominant in the northern/southern hemisphere. This pattern is opposite to the sign of magnetic helicity injected by differential rotation along east-west oriented polarity inversion lines, posing a major conundrum for solar physics. A resolution of this problem is offered by the magnetic helicity-condensation model of Antiochos. To investigate the global consequences of helicity condensation for the hemispheric chirality pattern, we apply a temporally and spatially averaged statistical approximation of helicity condensation. Realistic magnetic field configurations in both the rising and declining phases of the solar cycle are simulated. For the helicity-condensation process, we assume convective cells consisting of positive/negative vorticities in the northern/southern hemisphere that inject negative/positive helicity. The magnitude of the vorticity is varied as a free parameter, corresponding to different rates of helicity injection. To reproduce the observed percentages of dominant and minority filament chiralities, we find that a vorticity of magnitude $2.5 \times 10^{-6} \mathrm{~s}^{-1}$ is required. This rate, however, is insufficient to produce the observed unimodal profile of chirality with latitude. To achieve this, a vorticity of at least $5 \times 10^{-6} \mathrm{~s}^{-1}$ is needed. Our results place a lower limit on the small-scale helicity injection required to dominate differential rotation and reproduce the observed hemispheric pattern. Future studies should aim to establish whether the helicity injection rate due to convective flows and/or flux emergence across all latitudes of the Sun is consistent with our results.
\end{abstract}

Key words: Sun: activity - Sun: corona - Sun: magnetic fields

\section{Introduction}

Many phenomena found within the solar atmosphere exhibit hemispheric chirality patterns whose origin can be related to magnetic helicity. These patterns can be seen across a wide range of spatial scales and are found in sunspots, filaments, and coronal sigmoids, to name a few (see Zirker et al. 1997; Martin 2003, for reviews and additional phenomena). The exact strength of the hemispheric pattern varies among phenomena and can also depend on the objects' maturity. One such example is active regions that during the emergence phase have a weak hemispheric pattern of helicity, which subsequently strengthens as additional physical processes act on the emerged flux (see van Driel-Gesztelyi \& Green 2015, for a review and additional references). Understanding the origin, sign, and rates of magnetic helicity injection into the solar corona is key to explaining these and many additional phenomena that occur on the Sun. In terms of their hemispheric pattern, solar filaments are one of the most frequently studied phenomena as they trace the distribution and evolution of magnetic helicity from low to high latitudes and across a variety of magnetic field environments. Solar filaments and their birth grounds, filament channels (Gaizauskas 1998), are also key indicators of the location and concentration of free magnetic energy in the solar corona. As such, they are important for explaining eruptive phenomena such as coronal mass ejections (CMEs).

The hemispheric chirality pattern of solar prominences/ filaments describes the orientation of the dominant axial field along the main body of the filament, and it can be one of two types: dextral or sinistral (Martin et al. 1994). A dextral filament (or filament channel) is one in which the dominant axial magnetic field points to the right, as seen from an observer standing on the positive-polarity side of the polarity inversion line (PIL) and looking toward the PIL. From the same reference point, a sinistral filament has an axial magnetic field that points to the left. Filaments with dextral chirality dominate in the northern hemisphere, while those with sinistral chirality dominate in the southern hemisphere. One important aspect of this hemispheric pattern is that exceptions to the rule also exist. In terms of magnetic helicity, dextral filaments contain predominantly negative helicity; sinistral filaments have mainly positive helicity. A more detailed discussion of this hemispheric pattern is given in Section 2.

Previous studies of the origin of the hemispheric pattern of solar filaments have focused mostly on large-scale mechanisms for injecting magnetic helicity into the corona. Two mechanisms have been commonly considered: (i) differential rotation, which can inject both positive and negative magnetic helicity into each hemisphere (DeVore 2000), and (ii) the injection of helicity associated with the emergence of new active region magnetic bipoles. These large-scale bipoles are prescribed with an internal magnetic helicity that matches the observations of Pevtsov et al. (1995), namely negative/positive in the northern/southern hemisphere. The two processes described above work in conjunction with flux cancellation (surface diffusion), which helps to localize the helicity along the PIL over long periods of time. These mechanisms have been applied to a wide variety of situations, including idealized magnetic distributions (Mackay \& van Ballegooijen 2001, 2005) or observed distributions (Mackay et al. 2000; Yeates et al. 2007, 2008). In the longest-term study of this type, Yeates \& Mackay (2012) simulated the global corona for the entire length of cycle 23 , where in the rising phase, a good agreement with the hemispheric pattern was found. In contrast, in the declining phase, the simulation found that sinistral skew was dominant at high latitudes in the northern 
hemisphere and dextral in the southern hemisphere, which disagrees with observations.

While the mechanisms for helicity injection described above can be characterized as global mechanisms, Antiochos (2013) more recently developed a new concept for the local injection of magnetic helicity into the solar corona by small-scale convective motions and/or small-scale flux emergence as in the so-called magnetic carpet (Schrijver et al. 1997). This helicity is subsequently expected to condense onto PILs. In this helicity-condensation model, filament channels form through a three-stage process of helicity injection, transfer, and condensation that acts on the chromospheric and coronal magnetic fields. The chirality of the axial field and helicity at the PIL depend upon the associated sign and strength of the effective vortical motions due to the small-scale injection processes. If these effective vortical motions are mainly counterclockwise/ clockwise in the northern/southern hemisphere, then the resultant helicity injection is negative/positive, leading to dextral/sinistral filament channels. High-resolution numerical simulations (Knizhnik et al. 2015, 2017a; Zhao et al. 2015) have confirmed the accumulation of helicity at the perimeter of a compact region of such vortical flows in a plane-parallel corona, supporting the validity of the three-stage process described above. In a more definitive study of helicity condensation, in a bipolar magnetic region with a true PIL at the solar surface, Knizhnik et al. (2017b) formed a filament channel having concentrated shear and dipped field lines, as observed on the Sun.

A critical element of the helicity-condensation model is the magnitude and coherence of the vorticity of the underlying convective cells that drive the small-scale helicity injection. Its sign, strength, and distribution determine the axial field and helicity at the PIL. At present, these properties have not been firmly established from observations. However, evidence of the hemispheric dependence of the vorticity with sign dependence as described above has been found in the downflow lanes between cells (Duvall \& Gizon 2000; Gizon \& Duvall 2003; Komm et al. 2007). These studies indicate that the magnitude of the vorticity is on the order of $1 \times 10^{-6} \mathrm{~s}^{-1}$. Therefore the currently measured value of vorticity is comparable to the peak in the gradient of differential rotation, $\omega_{\mathrm{dr}}=0.85 \times 10^{-6} \mathrm{~s}^{-1}$, which occurs at $\lambda=54^{\circ}$ latitude (Snodgrass 1983). Assuming that the magnitude and coherence of the vortical motions can be established, the helicity-condensation mechanism has an advantage over the injection of magnetic helicity into the solar corona through the emergence of new active region bipoles, as the latter occurs only sporadically and at low latitudes. When eruptions occur (Yeates \& Mackay 2009) and this magnetic helicity is partially or fully lost, it cannot be regenerated in situ. In contrast, it is believed to be continually injected for the helicity-condensation model.

In Mackay et al. (2014), we considered the process of helicity condensation within the context of large-scale magnetic flux-transport simulations and global-scale modeling of the coronal magnetic field. To simulate the small-scale injection of magnetic helicity, a large-scale temporally and spatially averaged statistical approximation for the small-scale dynamics was developed. In this paper we refer to that approach as the statistically averaged helicity-condensation (SAHC) model. The consequences of this statistically averaged approach to helicity condensation, in cooperation or competition with other flux-transport processes, was considered in simple idealized magnetic field configurations. These configurations included high-latitude east-west-oriented polar crown or sub-polar crown PILs and the interaction of a single large-scale magnetic bipole with an axisymmetric polar field in both the rising and declining phases of the solar cycle.

The simulations demonstrated that to overcome the effect of differential rotation, which injects the incorrect sign of helicity in each hemisphere at east-west PILs, the vorticity of the supergranular cells had to be roughly 3-5 times higher than the local value of the differential-rotation gradient. This factor of 3-5 is required so that the supergranular helicity injection can overcome differential rotation not just locally at the PIL, but also in an extended zone around it. This is necessary because surface diffusion converges field lines with a large latitudinal footpoint separation toward the PIL. The latitudinal separation in footpoints means that the field lines experience a higher rate of differential rotation than that found locally at the PIL. This demonstrated the importance of both local (differential rotation, helicity condensation) and nonlocal (surface diffusion) processes on the accumulation of helicity.

The objective of the present paper is to advance our previous study in Mackay et al. (2014) to quantify the impact of helicity condensation as expressed by the SAHC model on the formation of solar filament channels in realistic, observationally derived magnetic field configurations that are representative of the solar cycle. Two periods of 16 Carrington rotations (CR) each, one in the rising and the other in the declining phase of the cycle, are selected. This allows us to examine the consequences of helicity condensation for the chirality of the magnetic field before and after the reversal of the polar fields. The paper is structured as follows. In Section 2 a review of observations of the hemispheric pattern of filaments is given. In Section 3 the global nonlinear force-free field evolutionary model is described along with the large-scale model for helicity condensation. In Section 4 the rising- and declining-phase periods are selected and the method of simulating the evolution of the radial magnetic field at the photosphere is described. The results for the two phases of the solar cycle under consideration are described in Sections 5 and 6 for various rates of helicity injection. Finally, in Sections 7 and 8 the discussion and conclusions are given, along with a description of future studies.

\section{Hemispheric Pattern of Filaments}

The observational study of Martin et al. (1994) was the first to quantify the existence of a hemispheric pattern for quiescent filaments. Using high-resolution $\mathrm{H} \alpha$ images of 72 quiescent filaments taken during the months of 1991 September and 1992 June, the authors found that $100 \%$ of the quiescent filaments in the northern hemisphere were of dextral chirality, while $72 \%$ in the southern hemisphere were of sinistral chirality. This agreed with earlier direct magnetic field measurements made within polar crown filaments by Rust (1967) and Leroy et al. (1983, 1984). While Martin et al. (1994) established the existence of a hemispheric pattern for quiescent filaments, their results showed no such pattern for active region filaments.

Following this, Pevtsov et al. (2003) performed a longerterm analysis of filament chirality over a two-year period from 2000-2001 in solar cycle 23. In contrast to the study of Martin et al. (1994), Pevtsov et al. (2003) used lower-resolution fulldisk observations of filament barbs from Big Bear Solar Observatory to determine the chirality. Their sample involved 


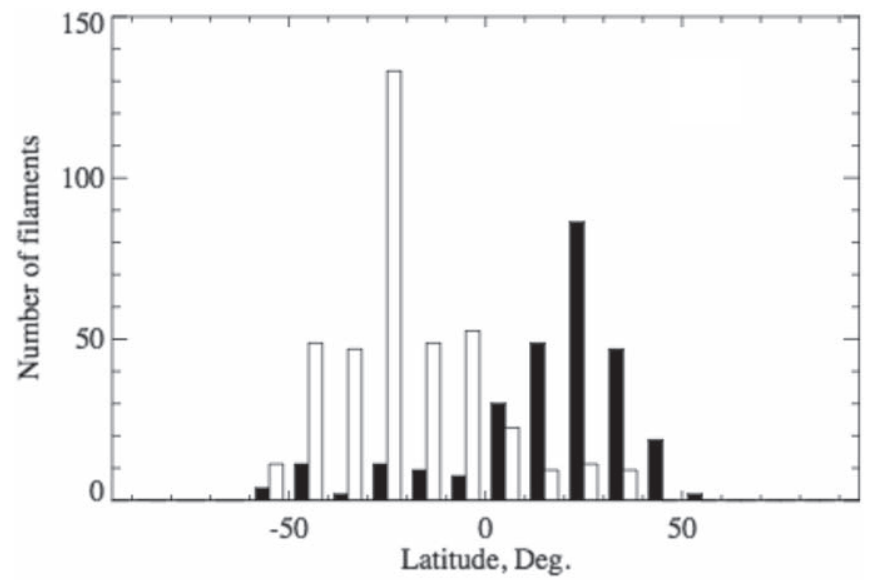

(a)

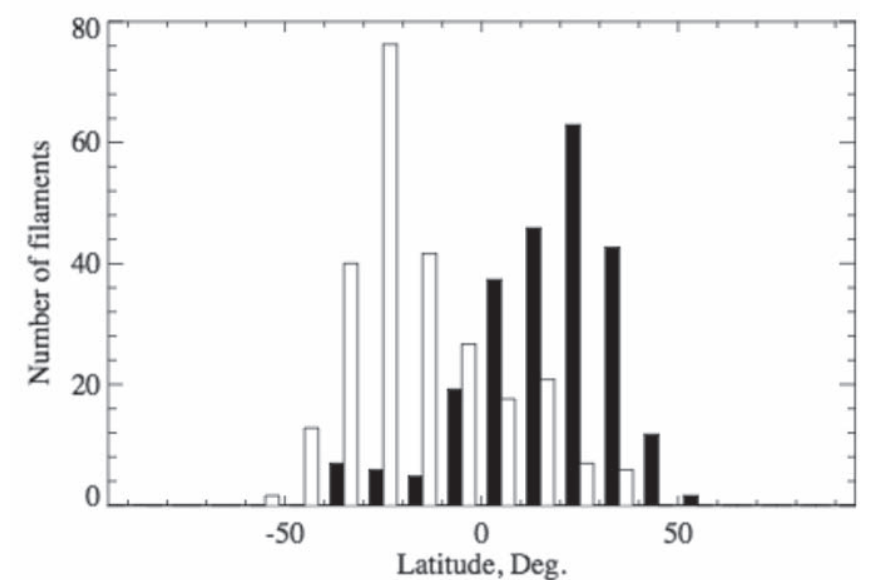

(b)

Figure 1. Results from Figure 6 of Pevtsov et al. (2003) showing the hemispheric distribution of dextral filaments (black histograms) and sinistral filaments (white histograms) for solar cycle 23 during the periods of (a) CR 1958-1972 (2000-2001) and (b) CR 1976-1985 (2001).

2310 determinations of chirality, therefore significantly higher numbers compared to Martin et al. (1994). More determinations of chirality were made as the study was conducted over a longer time period, and individual filaments may have been counted multiple times as they were not tracked during their disk passage. Through this study, the authors determined that $80.2 \%(85.5 \%)$ of quiescent filaments were dextral (sinistral) in the northern (southern) hemisphere, in overall agreement with Martin et al. (1994). In contrast to the results of Martin et al. (1994), Pevtsov et al. (2003) found that active region filaments also exhibit a hemispheric pattern, albeit slightly weaker, with $75 \%(76.7 \%)$ determined as dextral (sinistral) in the northern (southern) hemisphere. The reason for this disagreement with Martin et al. (1994) is unclear. The combined results of these studies of both quiescent and active region filaments are that in each hemisphere, $75 \%-80 \%$ of filaments exhibit dominant chirality.

While considering absolute numbers is one way of describing the hemispheric pattern, Figure 1 shows the latitudinal distribution of chirality (black $=$ dextral, white $=$ sinistral) for quiescent filaments taken from Pevtsov et al. (2003). The results are separated into periods (a) before polar field reversal (CR 1958-1972) and (b) after polar field reversal (CR 1976-1985). Both time periods show a similar profile, where in each hemisphere the dominant chirality peaks around $\pm 30^{\circ}$ latitude.
The authors note that there is a larger fraction of minority chirality in each hemisphere in the declining phase when the pattern may not be as strong. A similar distribution can be seen in Figure 9 of Martin et al. (1994).

\section{Global Nonpotential Evolutionary Model with Helicity Condensation}

To simulate the long-term, global evolution of the Sun's large-scale magnetic field (Mackay \& Yeates 2012), a global nonpotential evolutionary model is applied. In this model the Sun's magnetic field,

$$
\boldsymbol{B}=\left(B_{r}, B_{\theta}, B_{\phi}\right)=\nabla \times \boldsymbol{A},
$$

evolves through a continuous series of quasi-static nonlinear forcefree states as a consequence of boundary motions and magnetic flux emergence. To specify the continuously evolving photospheric boundary condition, a magnetic flux transport model is used (Sheeley 2005), while the response of the corona to these motions is modeled with a magneto-frictional relaxation technique (van Ballegooijen et al. 2000; Mackay \& van Ballegooijen 2006a).

The magnetic flux transport simulations describe the evolution of the radial magnetic field at the photosphere, $r=R_{\odot}$, over long periods of time under the combined effects of differential rotation (Snodgrass 1983), meridional circulation (Duvall 1979), and magnetic flux emergence. In addition, the magnetic field is subject to a random walk by small-scale flows such as supergranulation (Leighton 1964), represented as surface diffusion. The magnetic flux transport equation can be expressed in terms of the magnetic vector potential tangent to the solar surface, $\boldsymbol{A}_{s}$,

$$
\frac{\partial \boldsymbol{A}_{s}}{\partial t}=\boldsymbol{v}_{s} \times \boldsymbol{B}_{r}-D \nabla_{s} \times \boldsymbol{B}_{r} .
$$

Here $v_{s}$ is the surface velocity field including differential rotation and meridional circulation, whose assumed functional forms and parameters are given in Section 2.1 of Mackay et al. (2014), $D$ is the supergranular diffusion coefficient, $\boldsymbol{B}_{r}=\hat{\boldsymbol{r}} B_{r}$ is the radial component of the magnetic field at the photosphere, and $\nabla_{s}$ is the curl operator including only the tangential (latitude and longitude) components.

Within the coronal volume, which extends to $2.5 R_{\odot}$, the magnetic field evolves in response to the above motions at the photosphere through the ideal induction equation,

$$
\frac{\partial \boldsymbol{A}}{\partial t}=\boldsymbol{v} \times \boldsymbol{B}+\boldsymbol{H}_{s g},
$$

where $\boldsymbol{v}(\boldsymbol{r}, t)$ is the plasma velocity and $\boldsymbol{H}_{s g}$ represents a statistically averaged, large-scale representation of the process of helicity condensation introduced by Antiochos (2013). The time integration of the induction equation is carried out in the Weyl gauge. Because the plasma beta is low in the corona and we are primarily interested in the long-lived structures of the field (not in high-frequency dynamics such as magnetohydrodynamic, MHD, waves), we expect the coronal magnetic field evolution to closely follow a sequence of quasi-steady forcefree states. This is supported by the fact that the photospheric boundary motions described above are very slow compared to the coronal Alfvén speed. Therefore, we use the magnetofrictional method as in Yang et al. (1986) to capture the essential evolution, where the coronal plasma velocity is 
given by

$$
\boldsymbol{v}=\frac{1}{\nu} \frac{\boldsymbol{j} \times \boldsymbol{B}}{B^{2}}+v_{o} e^{-\left(2.5 R_{\odot}-r\right) / r_{w}} \hat{\boldsymbol{r}}
$$

and $\boldsymbol{j}=\nabla \times \boldsymbol{B}$. The first term on the right side reflects the fact that in the corona the Lorentz force is dominant (low beta condition). The effect of this "frictional" term is that when any field departs from a force-free state-as a result of boundary driving - the magnetic forces in the corona act to return the field to a force-free state (generally, a nonlinear force-free field). The second term represents a radial outflow velocity that is imposed to ensure that the field lines remain radial at the source surface $\left(r=2.5 R_{\odot}\right)$. In a crude manner, this outflow velocity simulates the effect of the solar wind in opening coronal field lines. Parameters are chosen such that its peak value is $v_{o}=100 \mathrm{~km} \mathrm{~s}^{-1}$ and its exponential fall-off length from the outer boundary is $r_{w}=0.1 R_{\odot}$. This term is negligible in the low closed-field corona. Full details of the numerical grid used to carry out the calculations along with the applied boundary conditions can be found in Section 2.1 of Mackay et al. (2014).

The final term in Equation (3) is a large-scale, temporally and spatially averaged, statistical approximation to the full helicity-condensation process of Antiochos (2013). This largescale representation is used in lieu of computationally resolving the flow fields on the size of supergranules and the fast reconnection dynamics that occur, which would be extremely challenging within a global simulation. This term is derived in the Appendix of our previous paper (Mackay et al. 2014), where it is shown that the average rate of injection of magnetic helicity into the large-scale coronal magnetic field, due to the small-scale processes such as the vortical motions associated with granular and supergranular convection cells, can be expressed as

$$
\boldsymbol{H}_{s g}= \begin{cases}\nabla_{r}\left(\zeta B_{r}\right), & r=R_{\odot} ; \\ 0, & \text { otherwise }\end{cases}
$$

In Equation (5), $\nabla_{r}$ is the gradient operator including only the radial derivative, and $\zeta$ parameterizes the rate and scale of the helicity injection at the photosphere. It has the dimensions of a diffusivity and takes the explicit form

$$
\zeta \equiv \overline{l^{2} \omega_{l}} / 2
$$

where $l$ and $\omega_{l}$ are the radius and angular rotation rate, respectively, within the convection cells. The overline denotes a spatial and temporal average over the characteristic scales of the convection. Hereafter, we refer to this large-scale approximation as the statistically averaged helicity-condensation (SAHC) model. The SAHC model is the only feasible approach at the present time for considering the effects of helicity condensation in competition with differential rotation, flux emergence, meridional flow, and surface diffusion over long periods of time. We note that this statistical approximation is entirely analogous to similar approximations made within the global diffusion model underlying the flux-transport equation for the large-scale field.

The consequences of the SAHC model in conjunction with other large-scale helicity injection mechanisms are encapsulated in terms of two parameters, the angular rotation rate and the length scale of the convection cells, without having to resolve the cells. To specify these parameters, we take $\bar{l}=20,000 \mathrm{~km}$ as an average value, where the individual observed values range from 14,000 to $30,000 \mathrm{~km}$. The specification of the angular rotation rate is slightly more problematic as neither the average vorticity of supergranules nor its spatial dependence versus latitude has been firmly established from observations. The most compelling measurements come from applying time-distance and ring-diagram methods of helioseismology to global solar oscillations (Duvall \& Gizon 2000; Gizon \& Duvall 2003; Komm et al. 2007). These show that the vorticity at supergranular cells is positive (counterclockwise) in the downflow lanes between cells in the northern hemisphere, and correspondingly negative (clockwise) in the southern hemisphere. The signs are reversed in the upwelling centers of the cells, but the field dwells most of the time in the downflow lanes. This behavior of the signed vorticity is consistent with the influence of Coriolis forces on convection. The magnitude of vorticity is found to be on the order of $1 \times 10^{-6} \mathrm{~s}^{-1}$. Additional observations are needed to determine the vorticity of the supergranular convection cells more precisely; the value of vorticity is currently at the limit of observational detection. Consequently, in the present investigation, we consider for simplicity only a spatially uniform value in each hemisphere whose sign changes across the equator. Magnitudes are chosen to be in the range $\left|\overline{\omega_{l}}\right|=$ $1-10 \times 10^{-6} \mathrm{~s}^{-1}$. Positive values of vorticity are used in the northern hemisphere to inject negative helicity, and negative values are used in the southern hemisphere to inject positive helicity. With this assumption, the helicity parameter $\zeta$ ranges from roughly 200 to $2000 \mathrm{~km}^{2} \mathrm{~s}^{-1}$, which is on the same order of magnitude as the surface diffusion coefficient, $D=450 \mathrm{~km}^{2} \mathrm{~s}^{-1}$. All simulations discussed within this paper are described in terms of the vorticity magnitude applied within the SAHC model, as it is a parameter that in principle can be observationally determined.

Because the vortical motions at the photosphere twist only the footpoints of the coronal magnetic field, the effect is to induce a horizontal twist component into the magnetic field at the base of the corona. After being injected at the base, this twist component propagates upward along the field lines through the ideal convection term $\boldsymbol{v} \times \boldsymbol{B}$ in Equation (3). Details of the numerical implementation of this term can be found in Section 2.2 of Mackay et al. (2014).

\section{Observational Input Data}

\subsection{Selection of Simulation Periods}

To investigate the global long-term consequences of helicity condensation in realistic magnetic field configurations, two simulation periods are selected. They represent the varying magnetic field configurations that occur on the Sun over the full solar cycle. One period is in the rising phase of the solar cycle, the other in the declining phase. This allows an investigation of the effect of the helicity-condensation process on the chirality of the global magnetic field before and after the reversal of the polar fields near cycle maximum using the SAHC approach. The evolving sets of PILs that result represent those typical of a full solar cycle.

The rising-phase period extends from 1997 December 11 to 1999 February 22 (436 days) between CRs 1930 and 1946. The 


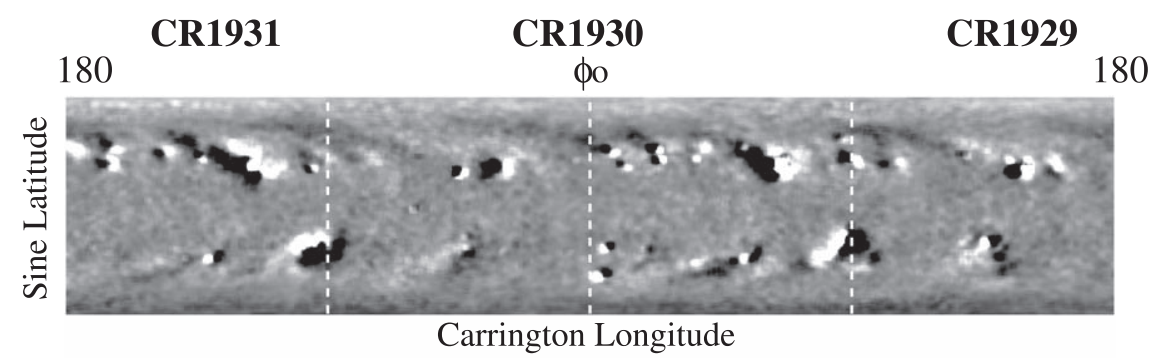

(a)

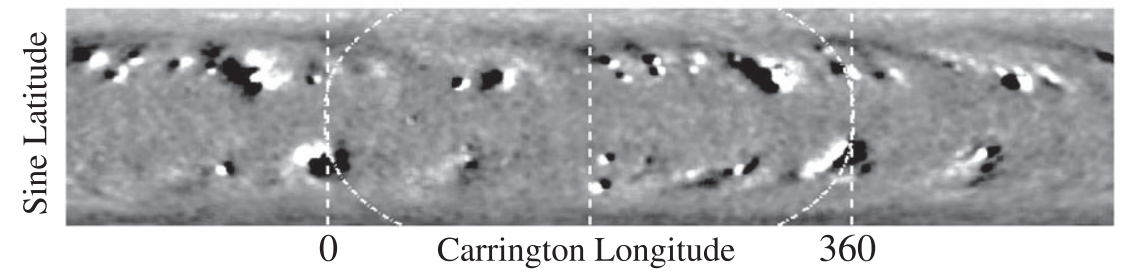

(b)

Figure 2. Illustration of the technique applied to produce an approximation to the instantaneous radial magnetic field globally on the Sun on 1997 December 11 corresponding to Carrington longitude $\phi_{O}$ at central meridian. (a): Three successive CRs (CR 1929-1931) joined together. The dashed lines denote the central meridian Carrington longitude on this day and the boundary lines between the three maps. (b): Synoptic magnetograms corrected for differential rotation about longitude $\phi_{O}$. The dash-dotted white lines denote the deformation of the boundary lines given in panel (a). The vertical dashed lines now give the boundary of the corrected radial magnetic field that is used to construct the initial potential field.

declining-phase period extends from 2003 February 6 to 2004 April 17 (437 days) during CRs 1999 through 2015. Each period covers 16 CRs so that there is sufficient time to study the development of nonpotential fields and their transport across the solar surface. While we are simulating observed periods, in the present paper, we do not carry out detailed one-to-one comparisons with observations on any given day. Instead, we consider characteristic and statistical properties of the chirality found along different orientations of the PILs that are typical of the rising and declining phases of the cycle. As a consequence, when we refer to the simulation results in discussions, we only describe the number of days of evolution, not the corresponding calendar date.

The rising-phase period simulates an interval of increasing magnetic activity, magnetic flux, and complexity in the coronal field. In contrast, the declining-phase period exhibits decreasing activity and flux overall. Both periods are chosen to be at least two years away from the reversal of the polar fields so that the polar fields are fully formed. For the data-driven simulations presented here, the CR magnetograms are used in two ways. First, they are used to produce an initial condition of the photospheric field along with an extrapolated potential coronal magnetic field (Section 4.2 and Appendix A). Second, they are used to determine where new large-scale magnetic flux emerges throughout the time period of each simulation (Section 4.3 and Appendix B). Including the flux of newly emerging active regions ensures that the simulated evolution of the photospheric field over these long periods of time is representative of the evolution observed on the Sun.

\subsection{Initial Condition}

For each of the rising- and declining-phase simulations, an initial photospheric field distribution is specified and a potential magnetic field constructed. Unfortunately, no complete observations exist of the Sun's global field on a given day, as only one side may be viewed at any time. While synoptic magnetograms do provide a global representation, they exhibit a time evolution extending over 27.3 days and cannot be directly used as the initial condition. Instead, we produce a best representation of the photospheric field on a given day by correcting the observed synoptic magnetograms for differential rotation. Figure 2 illustrates this process for the rising-phase simulation; full details can be found in Appendix A. The initial photospheric field then is evolved forward in time to approximately reproduce the magnetic field configurations occurring at later dates.

\subsection{Determination and Insertion of New Active Region Bipoles}

For the simulations to remain representative of what occurred on the Sun, new bipolar active regions matching the properties of new emerging regions on the Sun must be added. To determine the properties of the new active regions a semiautomated procedure is applied. Details of this procedure are given in Appendix B. In both the rising- and declining-phase simulations, a significant amount of new magnetic flux emerges. For the rising-phase simulation, 187 bipoles are identified over 436 days and add a total flux of $1.3 \times 10^{24} \mathrm{Mx}$ $\left(\approx 3.0 \times 10^{21} \mathrm{Mx}\right.$ day $\left.^{-1}\right)$. In the declining-phase case, 186 bipoles with a total flux of $1.2 \times 10^{24} \mathrm{Mx}\left(\approx 2.7 \times 10^{21} \mathrm{Mx}\right.$ day $\left.^{-1}\right)$ are inserted over 437 days. Nearly four times as much flux emerges throughout each simulation compared to the initial condition, hence the evolution of the global field is dominated by the emergence and transport of new active region bipoles.

The emerging bipoles determined from the synoptic magnetograms are included in the simulations through the insertion of idealized magnetic bipoles whose properties match those of the observed regions. The functional form for the magnetic bipoles along with details of the insertion technique are given in Appendix B. Due to the lack of information about when the bipoles actually emerged, with half expected to have emerged on the far side, each bipole is emerged on its day of central meridian passage as determined from the synoptic magnetograms. This means that the simulations only produce 
an approximate representation of the true magnetic field on the Sun at any given time. Therefore, we do not carry out a detailed one-to-one comparison with observations; rather, we carry out an inter-comparison of simulations with and without helicity condensation and perform a long-term statistical analysis.

When new magnetic bipoles emerge in the present study, they are inserted without any self-helicity, but do have a mutual helicity with the surrounding fields. The inclusion of selfhelicity (negative/positive in the northern/southern hemisphere) was found to be critical for replicating the hemispheric pattern in previous studies (Yeates et al. 2008; Yeates \& Mackay 2012). We exclude it in the present study as we wish to investigate the competing effects of the helicity condensation and surface flux transport processes. The additional complexity of including the self-helicity of emerging active regions will be investigated in future studies.

\subsection{Comparison of the Rising-phase Photospheric Field with Observations}

To demonstrate the fidelity of our global simulations, Figure 3 compares the radial magnetic field distributions produced by the simulation with the original CR synoptic magnetograms. It should be noted that the simulation and CR synoptic magnetograms are fundamentally different. The radial magnetic field from the simulation (left column) represents a snapshot of the simulated global magnetic field at a given instant in time. This is produced as a consequence of the initial condition (Section 4.2), the emergence of new active region bipoles (Section 4.3), and the terms and profiles used in the flux transport equation (Section 3). In contrast, the CR synoptic magnetograms (right column) provide a 27.3-day time representation and do not represent a single instant in time. A detailed discussion on how to compare these two photospheric field distributions is given in Appendix C.

After taking into account the differences between the simulation and observations and carrying out a visual comparison of the location, orientation, and extent of strong flux concentrations, in addition to the paths of the large-scale PILs, we conclude that the simulation produces a good representation of the observed large-scale field. Results similar to those shown in Figure 3 are also found for the decliningphase simulation. For both simulation periods, the large-scale surface field remains representative of the Sun's over the periods under investigation, without having to be reset with observations. Therefore the surface field is sufficiently realistic that we may carry out an accurate investigation including statistical analysis of the consequences of magnetic helicity condensation as described by the SAHC model along realistic orientations of the PILs that occur in both the rising and declining phases.

\section{Rising Phase}

We now present results for the rising-phase simulation. Five separate cases are discussed where the evolution of the coronal field with and without helicity condensation is studied. When included, the SAHC model is described in terms of the vorticity of the supergranular convection cells. Four nonzero values are considered: $\omega_{l}=1 \times 10^{-6} \mathrm{~s}^{-1}, 2.5 \times 10^{-6} \mathrm{~s}^{-1}, 5 \times 10^{-6} \mathrm{~s}^{-1}$, and $10 \times 10^{-6} \mathrm{~s}^{-1}$. Positive values of vorticity are applied in the northern hemisphere and negative values in the southern. To facilitate the discussion, the sign dependence of the vorticity with hemisphere is not mentioned further.

\subsection{Evolution of Global Quantities}

Figure 4 shows the variation of global integrated quantities for the rising-phase simulation. The global quantities include (a) surface flux, (b) magnetic energy, (c) open flux, and (d) volume-integrated current-density magnitude $|\boldsymbol{j}|$. In each graph the black line shows the results for $\omega_{l}=0 \mathrm{~s}^{-1}$, the red line $\omega_{l}=2.5 \times 10^{-6} \mathrm{~s}^{-1}$, the blue line $\omega_{l}=5 \times 10^{-6} \mathrm{~s}^{-1}$, and the green line $\omega_{l}=10 \times 10^{-6} \mathrm{~s}^{-1}$. Results for $\omega_{l}=1 \times$ $10^{-6} \mathrm{~s}^{-1}$ are not shown, to allow individual lines to be distinguished more easily.

As the SAHC model does not alter the evolution of the surface field, only the black line is shown for the surface flux (Figure 4(a)). There is a general trend toward increasing flux over time, with the final flux values approximately $30 \%$ higher than those at the start. The volume-integrated magnetic energy (Figure 4(b)) shows a similar trend to that of the surface flux. Values ranging from $2 \times 10^{33}$ to $7 \times 10^{33}$ erg occur, where increases in energy due to sporadic flux emergence can be clearly seen. There are clear differences in energy between the cases with and without helicity condensation. When helicity condensation is included, the energy is systematically higher at all times. The highest energies are found for the highest rates of vorticity and correspondingly helicity injection $\left(\omega_{l}=10 \times\right.$ $10^{-6} \mathrm{~s}^{-1}$ - green line). This clearly illustrates that the helicitycondensation process leads to a coronal field that is significantly more energized. For the highest rate of vorticity plotted $\left(\omega_{l}=10 \times 10^{-6} \mathrm{~s}^{-1}\right.$, green line $)$, the magnetic energy is on average $1.5-2 \times 10^{33} \mathrm{erg}$ higher than the case where $\omega_{l}=0 \mathrm{~s}^{-1}$ (black line).

As $\omega_{l}$ varies, the open flux follows a roughly identical trend in all cases, with the curves mostly lying on top of one another. However, there are key differences in the number and intensity of sporadic enhancements in the open flux. These enhancements are more frequent and have higher values as $\omega_{l}$ increases. They are due to the ejection of magnetic flux ropes corresponding to filament channels out of the corona, once the ropes become too strong to be held down by the overlying arcades (Mackay \& van Ballegooijen 2006a, 2006b). This denotes the onset of non-equilibrium behavior within the corona, where the only equilibrium state available requires the flux rope to be ejected. More frequent and larger enhancements occur as $\omega_{l}$ increases because more stress and helicity are injected into the corona. Each flux-rope ejection removes both magnetic energy and helicity from the corona.

Finally, in Figure 4(d), the volume integral of $|\boldsymbol{j}|$ is shown, which provides a measure of the average "non-potentiality" of the coronal field. For all of the simulations, the initial value is zero as we start with a potential field. Over the first 40 days of evolution, there is a rapid increase as the corona evolves into a balance between shearing, relaxing, and ejecting flux ropes out of the corona. Each curve shows an upward trend over time due to increasing magnetic activity. As $\omega_{l}$ increases, higher values are obtained for the volume-integrated current. Overall, Figure 4 shows that the rate of helicity injection expressed through the vorticity in the SAHC model determines a number of characteristics in the behavior of the coronal field. In general, higher values of the vorticity lead to a more energized and dynamic corona. 


\section{Simulation}
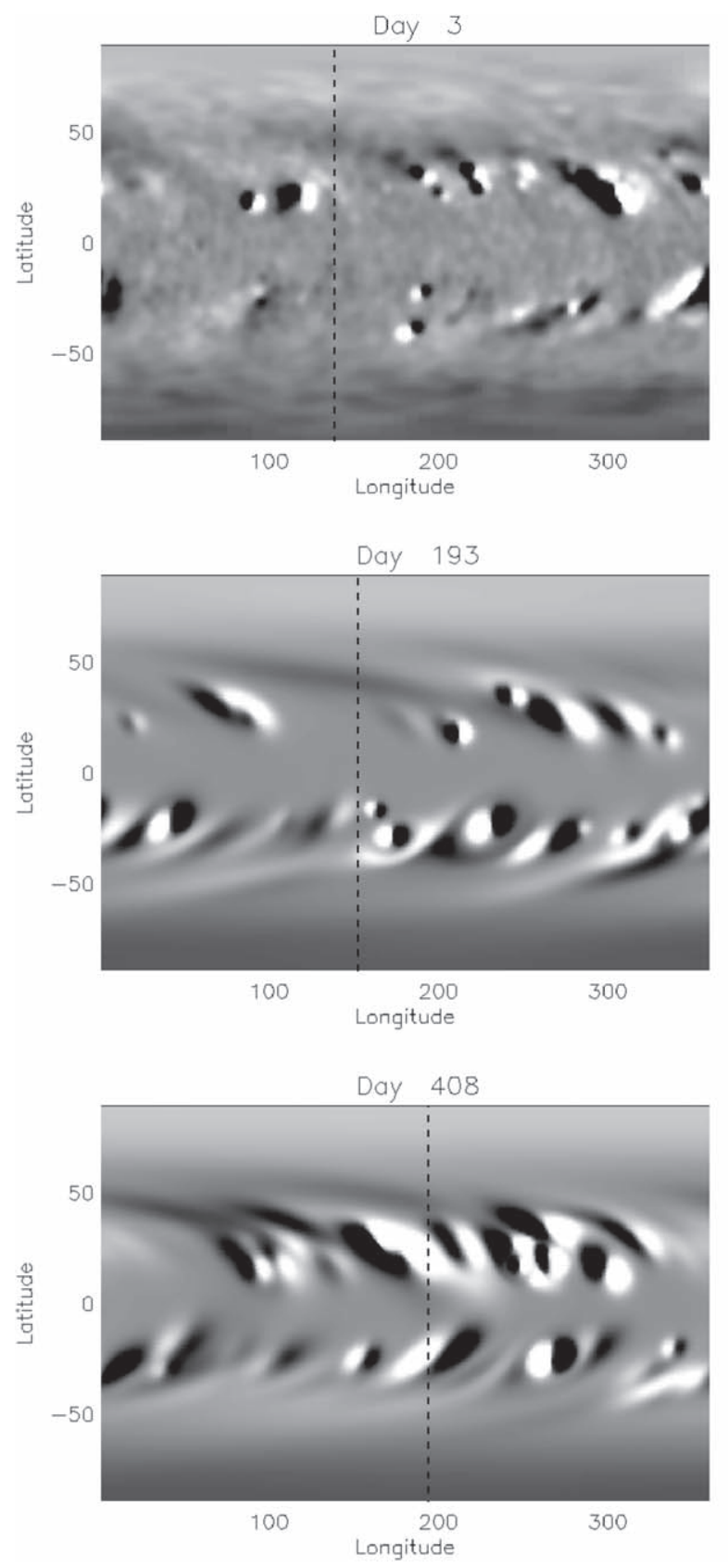

\section{Carrington Rotation Magnetograms}

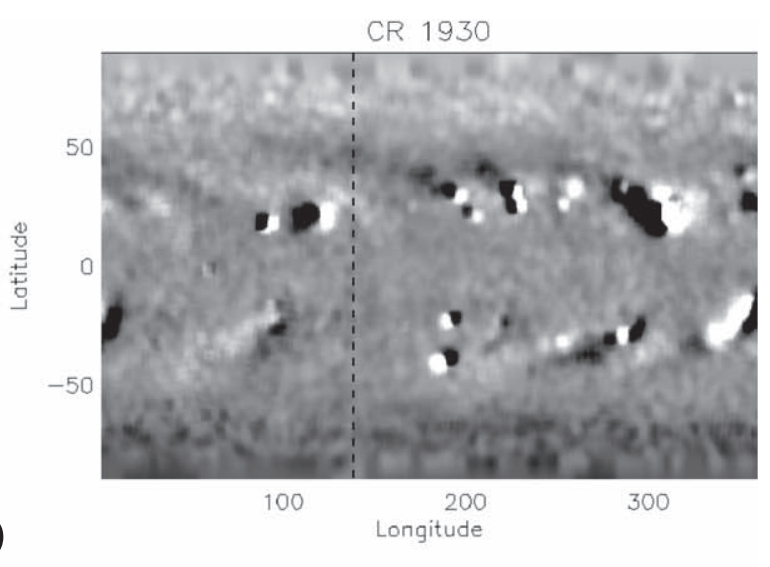

(a)

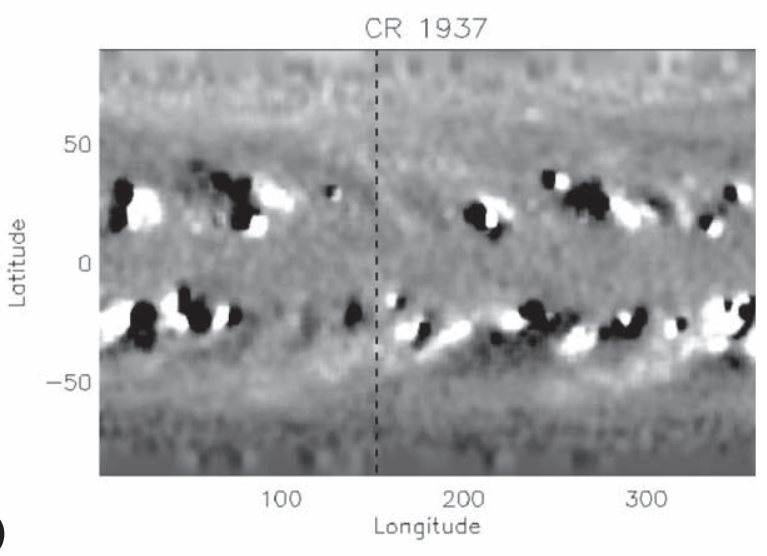

(b)

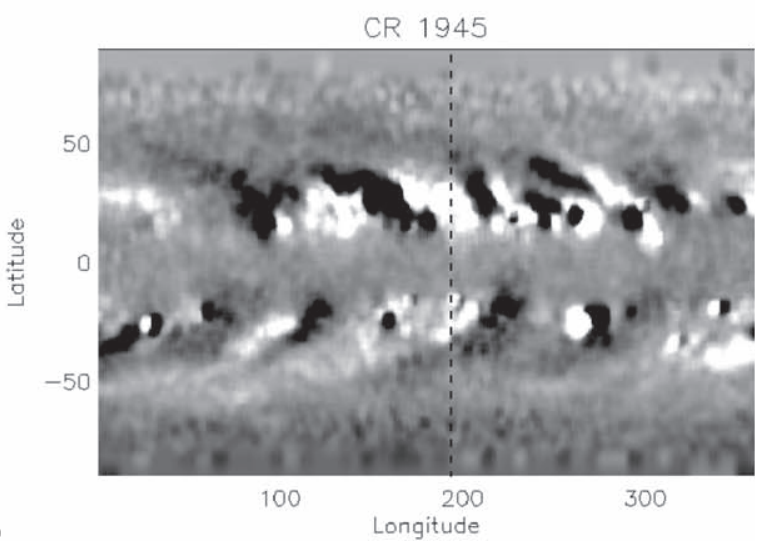

Figure 3. Comparison of the radial magnetic field in the rising-phase simulation with CR synoptic magnetograms. The left column shows the simulated result on (a) Day 3, (b) Day 193, and (c) Day 408, while the right column shows the corresponding smoothed synoptic magnetogram. The vertical dashed line in the plot denotes the longitude that lies at central meridian as seen from the Earth. The images are saturated at $\pm 20 \mathrm{G}$.

\subsection{Latitudinal Distribution of Skew}

To determine the latitudinal distribution of skew, the chirality is calculated in $13{ }^{\circ} .8$ latitude bins centered at the equator $\left(0^{\circ}\right.$ latitude), low latitudes $\left( \pm 13^{\circ} .8, \pm 27^{\circ} .7\right)$, mid latitudes $\left( \pm 41.5, \pm 55^{\circ} .4\right)$, and finally high latitudes $\left( \pm 69^{\circ} .2\right.$, $\left.\pm 83^{\circ} .1\right)$. Within each latitudinal bin, the chirality along all of the PILs enclosed by the bin is determined in terms of the skew $(\gamma)$ of the field. The skew is given by

$$
\cos \gamma=\frac{\hat{\boldsymbol{n}} \cdot \boldsymbol{B}_{s}}{|\hat{\boldsymbol{n}}|\left|\boldsymbol{B}_{s}\right|}
$$

where

$$
\hat{\boldsymbol{n}}=\frac{\nabla_{s} B_{r}}{\left|\nabla_{s} B r\right|}
$$

is the local normal to the PIL and $\boldsymbol{B}_{s}$ is the horizontal field passing over the PIL at a height of $10,000 \mathrm{~km}$. Dextral skew is defined as $\gamma>30^{\circ}$, sinistral skew as $\gamma<-30^{\circ}$, and weak skew as $|\gamma| \leqslant 30^{\circ}$. Once the skew is calculated along all of the PILs within each latitudinal bin, the total arc-length of the PIL that exhibits either dextral or sinistral skew within each 


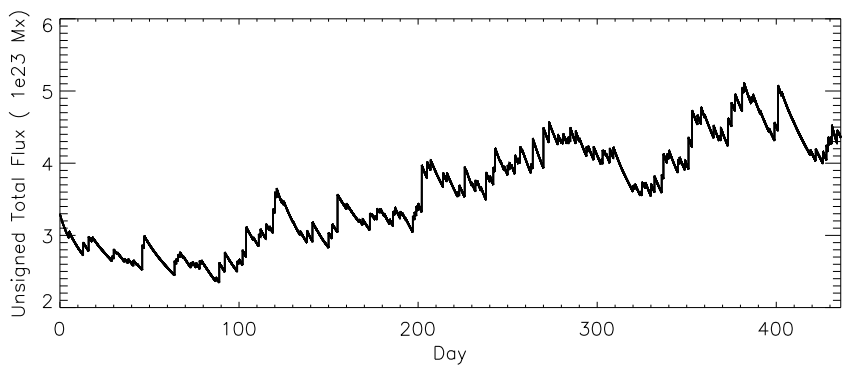

(a)

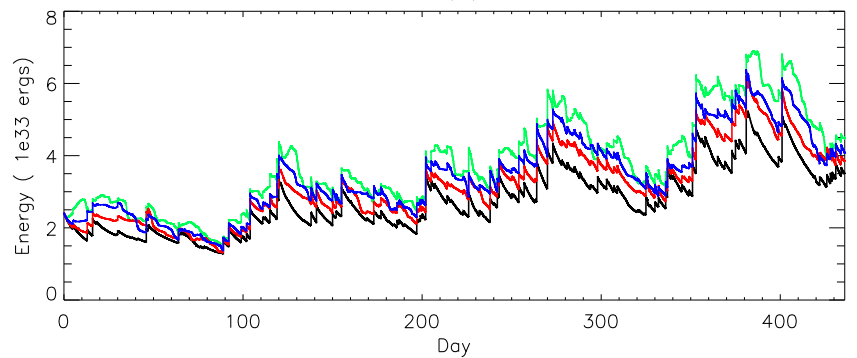

(b)

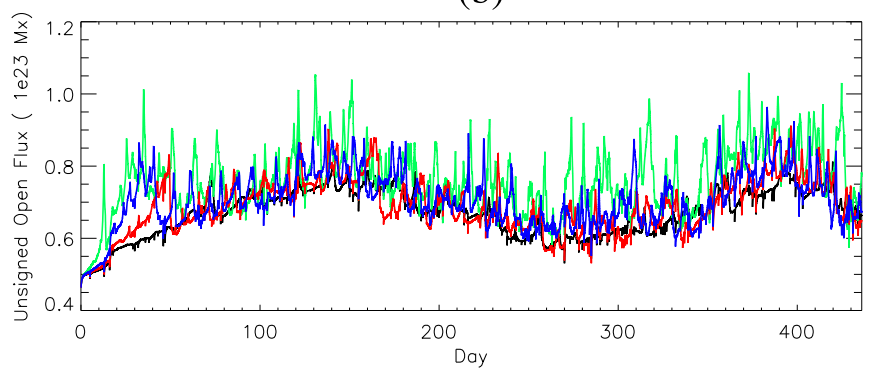

(c)

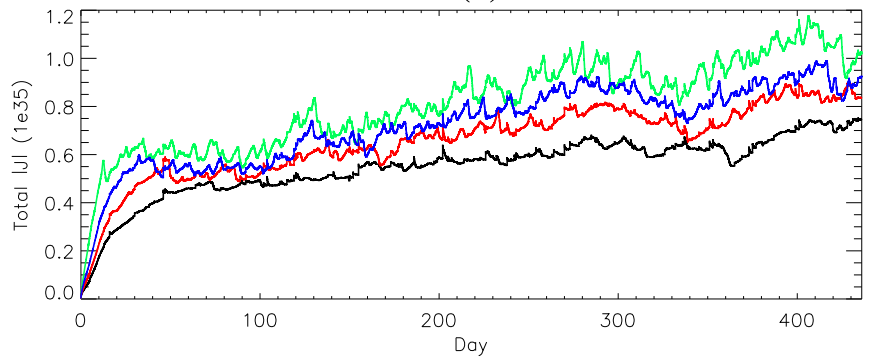

(d)

Figure 4. Properties of the global integrated quantities for the rising-phase simulation. Shown are (a) surface flux, (b) volume-integrated magnetic energy, (c) open flux, and (d) volume-integrated $|j|$ vs. time in days. Results are shown for simulations where $\omega_{l}=0 \mathrm{~s}^{-1}$ (black line), $\omega_{l}=2.5 \times 10^{-6} \mathrm{~s}^{-1}$ (red line), $\omega_{l}=5 \times 10^{-6} \mathrm{~s}^{-1}$ (blue line), and $\omega_{l}=10 \times 10^{-6} \mathrm{~s}^{-1}$ (green line).

latitudinal bin is calculated. The chirality is therefore expressed as the physical length of the PIL that exhibits either dextral or sinistral skew.

Figure 5 shows the distributions of the physical length of dextral (red) and sinistral (blue) skew, expressed in units of solar radii, versus latitude for the rising-phase simulations. Results are shown for the cases where (a) $\omega_{l}=0 \mathrm{~s}^{-1}$, (b) $\omega_{l}=2.5 \times 10^{-6} \mathrm{~s}^{-1}$, (c) $\omega_{l}=5 \times 10^{-6} \mathrm{~s}^{-1}$, and (d) $\omega_{l}=10 \times 10^{-6} \mathrm{~s}^{-1}$. Results for $\omega_{l}=1 \times 10^{-6} \mathrm{~s}^{-1}$ are not shown as they are very similar to the case $\omega_{l}=0 \mathrm{~s}^{-1}$. In each simulation, the flux emergence and transport parameters are identical, leading to exactly the same distribution and evolution of the radial field at the photosphere, and correspondingly, the total length of the PIL. The simulations are sampled once per 27 days (one CR) over the entire extent of the simulation, neglecting the first rotation. To produce the results that are displayed in each bin, the physical length of skew is averaged over the entire simulation. This minimizes any bias due to specific events, such as eruptions removing helicity and skew from the simulation. If no values are given, then there are no PILs within the latitudinal range enclosed by the bin.

Figure 5(a) presents the results when only surface flux transport and magnetic flux emergence act on the coronal field. No helicity injection from the SAHC model is included, so this simulation is the baseline case compared with the rest. It is clear that the skew along the PILs in each hemisphere exhibits an antisymmetric pattern across the equator, and that a bimodal distribution is produced. At latitudes within \pm 41.5 , the chirality visually matches that of the observed hemispheric pattern, with dextral/sinistral skew dominating in the northern/southern hemispheres. Although this pattern is clear, it is not as strong as is found within observations. At latitudes beyond $\pm 55^{\circ} .4$, the simulated chirality pattern reverses. This result is wholly inconsistent with the observed hemispheric pattern as currently understood and is caused by the action of differential rotation on east-west oriented PILs.

We now include the process of helicity condensation to determine whether a latitudinal pattern of skew consistent with the observed hemispheric pattern can be produced. In all cases at equatorial latitudes, there is no significant change in the physical length of sinistral and dextral chirality for any value of the vorticity. This is because at low latitudes, many field lines straddle the equator and have opposite signs of helicity injected at their two ends. This helicity propagates along field lines and cancels, providing no net effect. In contrast, when helicity injection is included at a rate of $\omega_{l}=1 \times 10^{-6} \mathrm{~s}^{-1}$ (not shown) or (b) $\omega_{l}=2.5 \times 10^{-6} \mathrm{~s}^{-1}$, at latitudes within $\pm 41^{\circ} .5$ the dominant skew in each hemisphere is strengthened. At latitudes beyond $\pm 55^{\circ} .4$, however, the skew remains inconsistent with the observed hemispheric pattern and the overall bimodal distribution persists.

To produce a chirality pattern that is consistent with current observations over all latitudes, the vorticity of the supergranular cells must be increased to $5 \times 10^{-6} \mathrm{~s}^{-1}$. At this value, the dextral/sinistral chirality is now dominant at all latitudes in the northern/southern hemisphere (Figures 5(c) and (d)). The distribution of each type of skew has a single peak occurring at low to mid latitudes where the pattern is strongest.

Observationally, the hemispheric pattern of filaments is often quoted as the relative number of dextral versus sinistral filaments in each hemisphere (Martin et al. 1994; Pevtsov et al. 2003). This number is approximately $75 \%: 25 \%$ between the majority and minority chiralities. The relative lengths of the PILs in each hemisphere that are categorized as having either dextral or sinistral skew are given in Table 1. The skewed portions of the PIL satisfy this observed ratio when a vorticity of $\omega_{l} \approx 2.5 \times 10^{-6} \mathrm{~s}^{-1}$ is applied. However, the correct latitudinal distribution is only seen for $\omega_{l}=5 \times 10^{-6} \mathrm{~s}^{-1}$. Further simulations show that the minimum value for the vorticity to achieve the correct percentage ratio and dominance at high latitudes is $\omega_{l} \approx 4.75 \times 10^{-6} \mathrm{~s}^{-1}$.

The analysis of the bar plots in the rising-phase simulations show that a dominant hemispheric pattern consistent with observations over a one-year timescale requires $\omega_{l} \approx 5 \times 10^{-6} \mathrm{~s}^{-1}$. At this vorticity, the SAHC model can counter differential rotation at high latitudes to match the chirality found in observations. One important aspect of the competing effects of differential rotation and helicity condensation is that as the vorticity increases between 

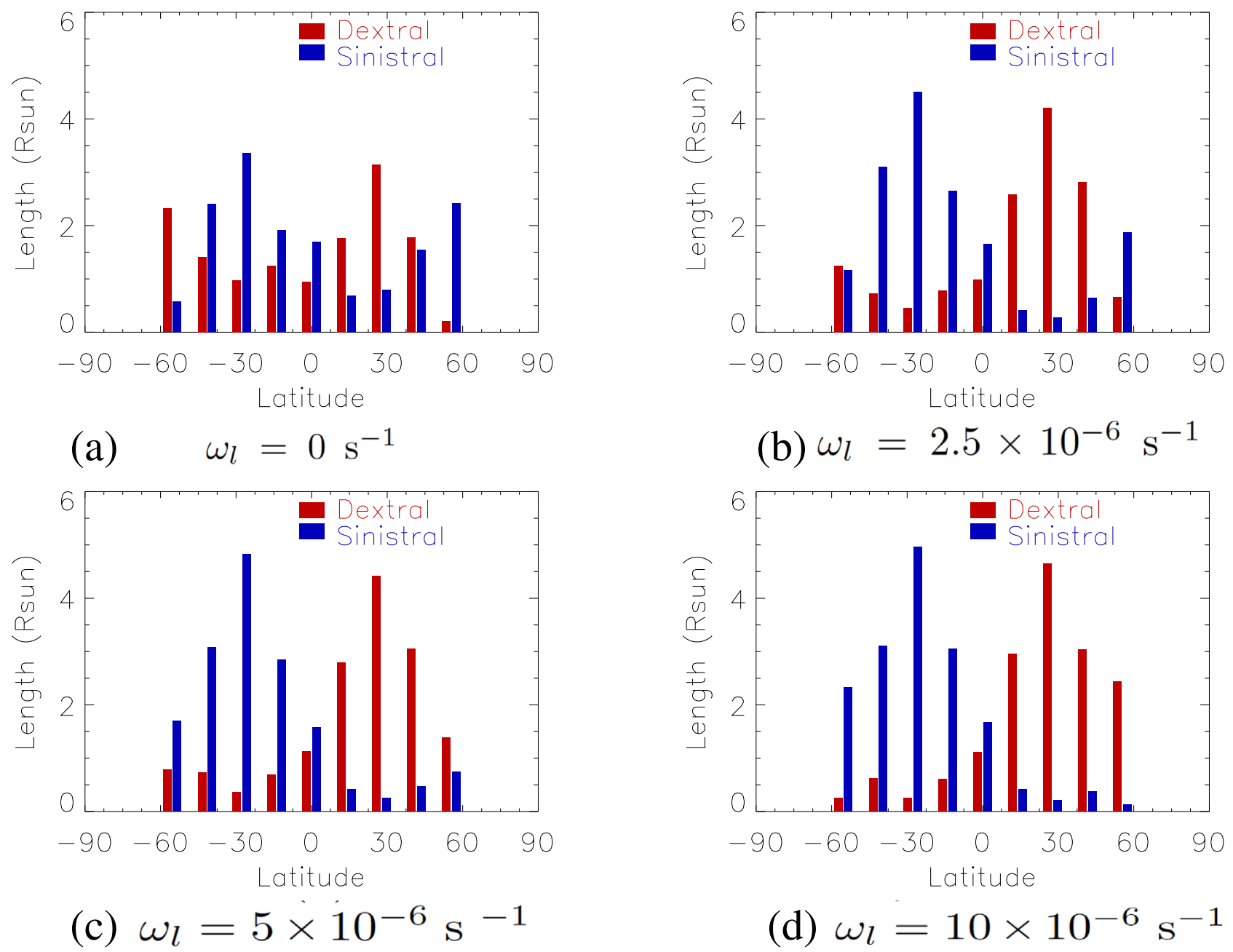

Figure 5. Bar plots showing the physical length of the PILs as a function of latitude that exhibit dextral (red) or sinistral (blue) chirality for the rising-phase simulation. The results are calculated in 13.8 wide latitudinal bins centered at latitudes of $\pm 0^{\circ}, 13.8,27^{\circ} .7,41.5,55^{\circ} .4,69^{\circ} .2$, and $83^{\circ} .1$, and the physical length of the PIL is given in units of solar radii. The graphs are shown for (a) $\omega_{l}=0 \mathrm{~s}^{-1}$ (i.e., surface flux transport only), (b) $\omega_{l}=2.5 \times 10^{-6} \mathrm{~s}^{-1}$, (c) $\omega_{l}=5 \times 10^{-6} \mathrm{~s}^{-1}$, and (d) $\omega_{l}=10 \times 10^{-6} \mathrm{~s}^{-1}$.

Table 1

Percentage Distributions of Dextral and Sinistral Skew in the Northern and Southern Hemispheres vs. the Vorticity $\omega_{l}$ Assumed for the Supergranular Helicity Injection in the Rising-phase Simulations

\begin{tabular}{lcc}
\hline \hline$\omega_{l}$ & $\begin{array}{c}\text { Northern } \\
\text { Dextral/Sinistral } \\
\%\end{array}$ & $\begin{array}{c}\text { Southern } \\
\text { Dextral/Sinistral } \\
\%\end{array}$ \\
$\mathrm{~s}^{-1}$ & $\% 6 / 44$ & $42 / 58$ \\
0 & $68 / 32$ & $30 / 70$ \\
$1 \times 10^{-6}$ & $76 / 24$ & $22 / 78$ \\
$2.5 \times 10^{-6}$ & $86 / 14$ & $17 / 83$ \\
$5 \times 10^{-6}$ & $92 / 8$ & $11 / 89$ \\
$10 \times 10^{-6}$ & & \\
\hline
\end{tabular}

values of $1-5 \times 10^{-6} \mathrm{~s}^{-1}$, the physical length of the sheared field lines at high-latitude PILs becomes less. In contrast, the fields at low latitude become more strongly sheared. Only after the vorticity exceeds $5 \times 10^{-6} \mathrm{~s}^{-1}$ does the length of shear increase at high latitudes. The reason for this behavior is that within the range $1-5 \times 10^{-6} \mathrm{~s}^{-1}$, the helicity-condensation process can just counter the sign and magnitude of helicity injected by differential rotation. Values greater than $5 \times 10^{-6} \mathrm{~s}^{-1}$ are required to both counter differential rotation and build up the opposite sign of helicity consistent with the hemispheric pattern in the coronal field.

\subsection{PIL Skew Distributions}

The evolution of the skew along individual PILs is shown in Figure 6. In each plot, white/black represents the photospheric flux distribution with the fields saturated at $\pm 20 \mathrm{G}$. The paths of the PILs at the level of the photosphere are also shown, color-coded according to the chirality of skew at each location. The skew is calculated at a height of $10,000 \mathrm{~km}$ : red denotes dextral skew, blue sinistral skew, and green weak skew, as defined in Section 5.2.

Results are shown for the early part of the simulations, on Days 28 (a) and (e) and 108 (b) and (f), and the later stages of the simulations, on Days 248 (c) and (g) and 383 (d) and (h). The baseline results for $\omega_{l}=0 \mathrm{~s}^{-1}$ are shown in panels (a)-(d); the results for $\omega_{l}=5 \times 10^{-6} \mathrm{~s}^{-1}$ are shown in panels (e)-(h). As the photospheric field and the paths of the PILs are identical, the two simulations can be compared directly. The only difference is the orientation of the field lines passing over the PILs.

Each simulation starts off on Day 1 with the same initial condition of a potential field, where the skew along the entire path of the PIL is weak. After approximately $1 \mathrm{CR}$, a significant amount of skew has been produced along the PILs in both simulations. In Figure 6(a), a clear latitudinal pattern of 


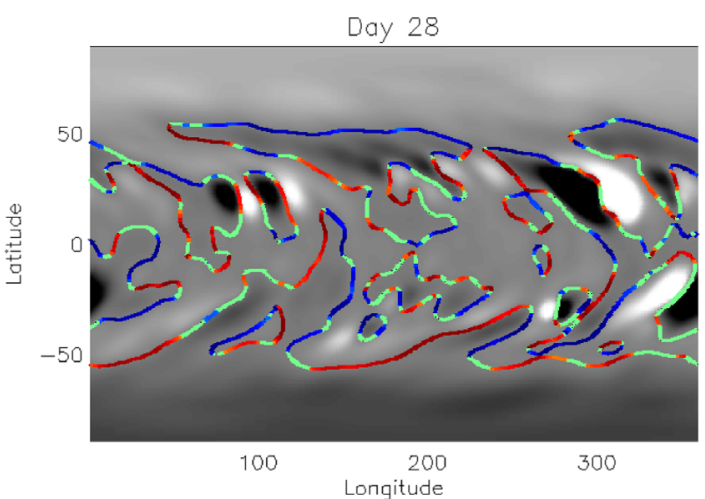

(a)

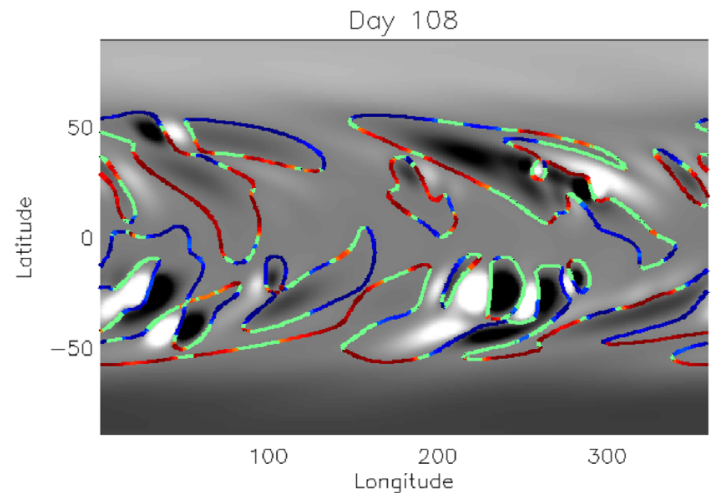

(b)

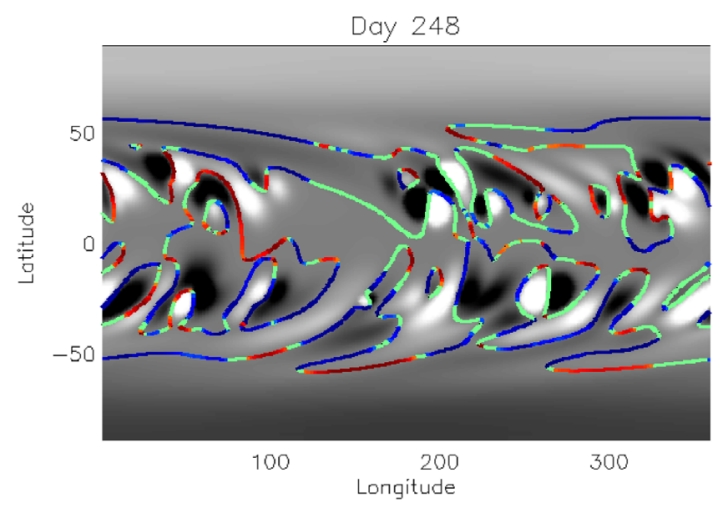

(c)

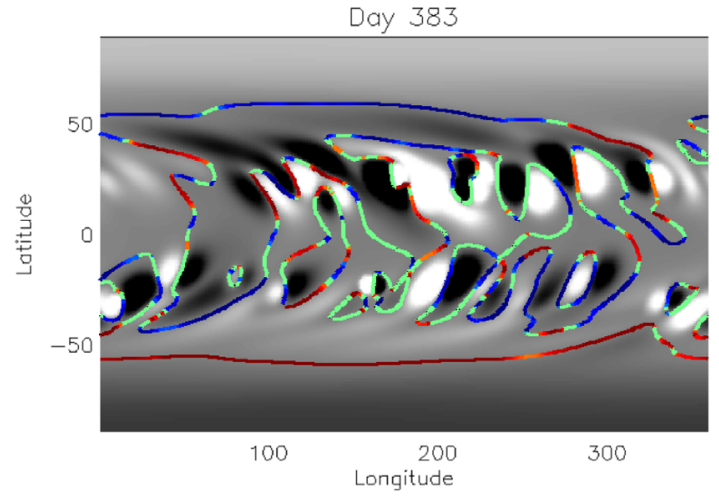

(d)

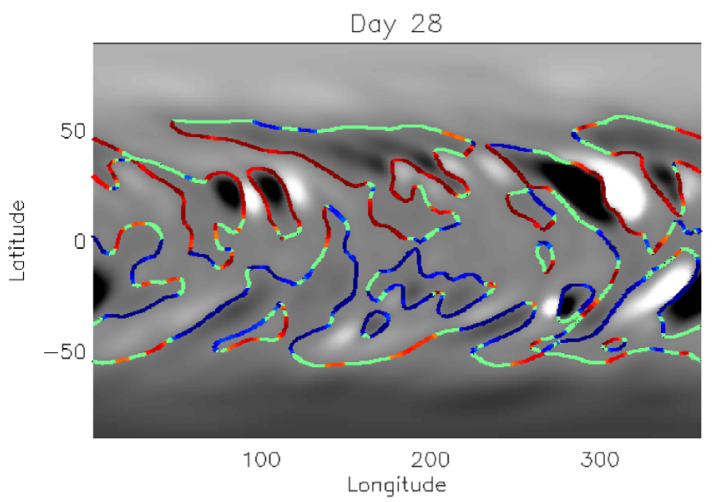

(e)

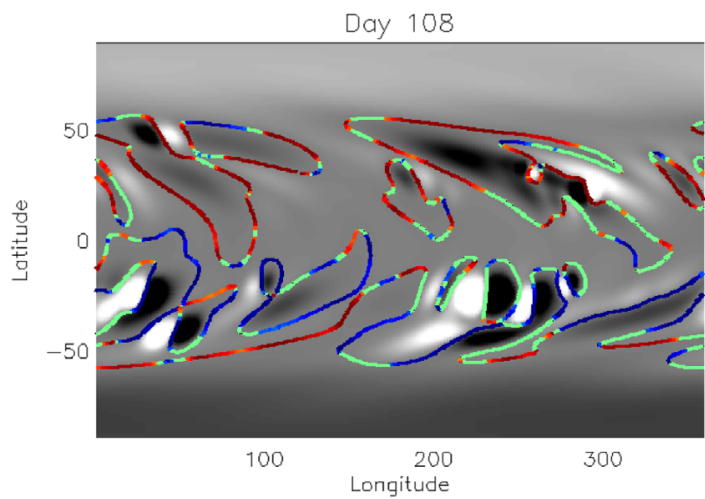

(f)

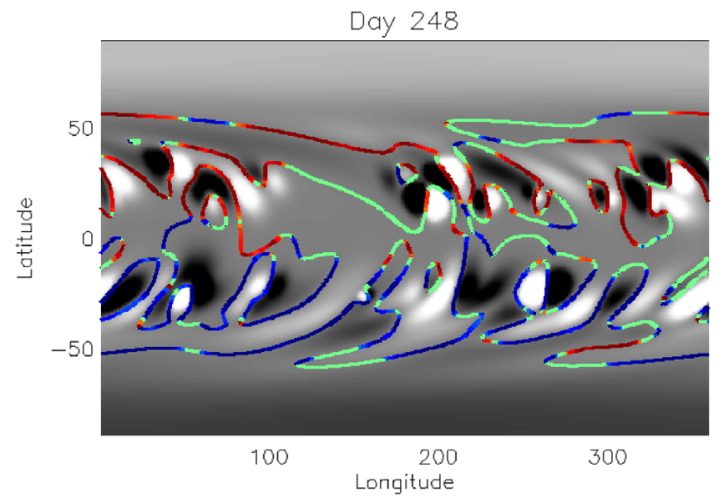

(g)

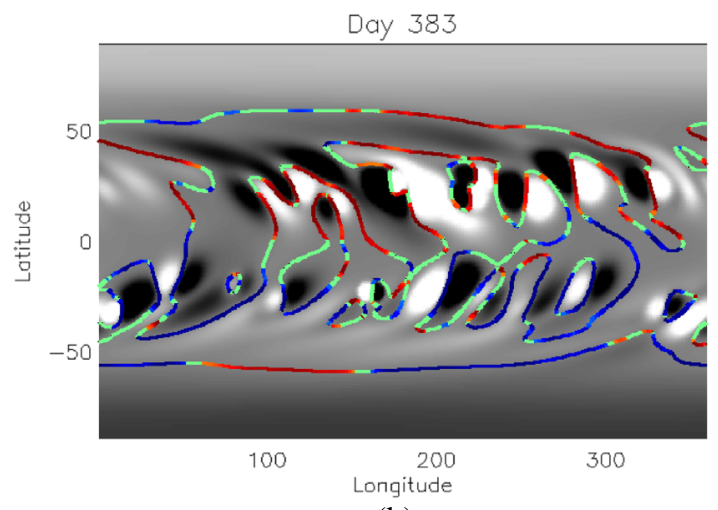

(h)

Figure 6. Chirality in the rising-phase simulation for (a)-(d) $\omega_{l}=0 \mathrm{~s}^{-1}$ and (e)-(h) $\omega_{l}=5 \times 10^{-6} \mathrm{~s}^{-1}$, on (a) and (e) Day 28, (b) and (f) Day 108, (c) and (g) Day 248, and (d) and (h) Day 383. In each plot, the path of the PIL is shown along with the chirality of the field that lies above it at a height of 10,000 km. Red denotes dextral chirality, blue sinistral chirality, and green weak chirality; the color bar at the right gives the relationship between the color along the PIL and the skew angle. White/black denotes the underlying positive/negative flux with the values saturated at $\pm 20 \mathrm{G}$. 
skew is forming. In general, along the north-south oriented PILs that lie at low to mid latitudes, dextral/sinistral skew is found in the northern/southern hemisphere (although exceptions to this are possible and do occur). In contrast, the opposite skew is found at mid to high latitudes, where along the eastwest oriented PILs sinistral/dextral skew is produced in the northern/southern hemisphere. This result is inconsistent with the observed hemispheric pattern of filaments. At all latitudes, the simulations have a significant amount of scatter and presence of weak skew.

The origin of this latitudinal pattern of skew can be understood straightforwardly as a consequence of differential rotation acting on north-south and east-west oriented PILs (Zirker et al. 1997). At low to mid latitudes, where the PILs in each hemisphere are predominantly oriented north-south, differential rotation injects negative/positive helicity into the overlying field lines in the northern/southern hemisphere (DeVore 2000). Negative helicity produces dextral skew, while positive helicity produces sinistral skew. On the other hand, at latitudes beyond $\pm 45^{\circ}$, the PILs are mostly aligned east-west and the sign of helicity injection by differential rotation reverses to be positive/negative in the northern/southern hemisphere and correspondingly produces sinistral/dextral skew. The bimodal pattern seen in Figure 5(a) is a consequence of differential rotation injecting both signs of helicity into the coronal field of each hemisphere according to the dominant local orientation of the PILs.

Throughout the entire simulation when $\omega_{l}=0 \mathrm{~s}^{-1}$, a similar pattern of skew is found along the PILs on Days 108, 248, and 383. By Day 383 (Figure 6(d)), a continuous polar crown PIL has formed in both the northern and southern hemispheres. The skew along this polar crown PIL is sinistral/dextral in the northern/southern hemispheres, inconsistent with current understanding of the hemispheric pattern of filaments. In the study of Yeates \& Mackay (2012), this inconsistency was overcome through inclusion of self-helicity in the emerging active region bipoles. We have neglected the self-helicity in this study in order to establish whether helicity condensation alone can reproduce the observed hemispheric pattern. For the simulations with $\omega_{l}=1 \times 10^{-6} \mathrm{~s}^{-1}$ and $\omega_{l}=2.5 \times 10^{-6} \mathrm{~s}^{-1}$, the skew along the individual PILs follows rather closely that already described for $\omega_{l}=0 \mathrm{~s}^{-1}$. Although the overall properties are the same, detailed differences do occur in the exact length and strength of the skew at any one time or location.

To examine further the consequences of helicity condensation in the SAHC model, Figures 6(e)-(h) show the skew of the field along the PILs when $\omega_{l}=5 \times 10^{-6} \mathrm{~s}^{-1}$ (similar results are found for $\omega_{l}=10 \times 10^{-6} \mathrm{~s}^{-1}$ ). By comparing Figure 6(e) with Figure 6(a), it is apparent that at low to mid latitudes in the northern/southern hemisphere, dextral/sinistral skew is generally much more dominant, although some exceptions still exist. The most significant differences between the two simulations occur at high latitudes, where along the east-west oriented PILs the helicity-condensation process has countered the helicity injected by differential rotation. This leads to a significant increase in the lengths of the PILs that have weak skew. As the simulation progresses to later times, similar differences can be seen at low to mid latitudes in both hemispheres. At high latitudes for $\omega_{l}=5 \times 10^{-6} \mathrm{~s}^{-1}$, there are many locations in both the northern and southern hemispheres where the chirality along east-west PILs has reversed compared to that found for $\omega_{l}=0 \mathrm{~s}^{-1}$. The dominant skew is now consistent with the observed hemispheric pattern.

By the end of the simulation period on Day 383 (Figure 6(h)), a large fraction of the sinistral skew in the north and dextral in the south has been removed. Nevertheless, some pockets of minority chirality remain. It is interesting to note that even with the high value of vorticity, which is over five times greater than the peak value in the gradient of differential rotation, some minority chirality is still produced in each hemisphere. In addition, significant lengths of the PILs have weak skew. The variation of the coronal field that leads to this pattern is considered through a specific example in Section 5.4.

There is a complex relationship between the local effects of differential rotation and helicity condensation that inject helicity and the nonlocal effects of surface diffusion that act to concentrate the helicity along the PIL. These processes were quantified through case studies in Mackay et al. (2014). Within our simulations, the skew along any one PIL changes continually as these combined effects act. In addition, abrupt changes in chirality and loss of helicity occur as flux ropes are ejected. These ejections can partially or fully remove the shear above the PILs, leading to reductions in the absolute amount of helicity.

To illustrate how these various terms change the chirality between the simulations with $\omega_{l}=0 \mathrm{~s}^{-1}$ and $\omega_{l}=5 \times$ $10^{-6} \mathrm{~s}^{-1}$, Figure 7 plots the chirality differences along the PILs. Six possible changes are quantified and color-coded in the figure. For simplicity, we discuss only the results for the northern hemisphere; the converse results hold in the southern hemisphere. In the northern hemisphere, the dominant change at low to mid latitudes is the strengthening of dextral skew (orange) and the weakening of sinistral skew (cyan). At other locations, the chirality is reversed from sinistral to dextral (dark red). All of these changes are consistent with the injection of additional negative helicity due to the SAHC model. On the other hand, there also are locations where dextral skew is weakened (yellow) or sinistral skew is strengthened (light blue). It is crucial to realize that although the SAHC model injects only negative/positive helicity into the northern/ southern hemisphere, a variety of changes in the field can occur when the additional local and nonlocal effects of differential rotation, surface diffusion, and flux-rope ejections are taken into account. At any one time and location, the chirality may not change strictly according to the sign of the helicity being injected by the SAHC model, relative to the case where no such injection occurs whatsoever.

\subsection{Evolution of Field Lines}

To illustrate the complex interplay of the helicity injection, localization, and removal processes, Figure 8 illustrates the evolution of field lines in the southern hemisphere along a single mid- to high-latitude PIL that lies between $\phi=\left[100^{\circ}\right.$, $\left.303^{\circ}\right]$ and $\lambda=\left[-60^{\circ}, 0^{\circ}\right]$. Results are shown for the simulation with $\omega_{l}=0 \mathrm{~s}^{-1}$ (a)-(d) and $\omega_{l}=5 \times 10^{-6} \mathrm{~s}^{-1}$ (e)-(h). In each plot, red represents positive flux, green negative flux, and dashed black lines the PILs. Blue lines are magnetic field lines passing over the PIL at a height of $10,000 \mathrm{~km}$. Results are shown on Days (a) and (e) 8, (b) and (f) 28, (c) and (g) 38, and (d) and (h) 48. Those for Day 28 correspond to the skew along the PILs shown in Figures 6(a) and (e). In Figures $8((\mathrm{a})$ and (e)), four specific locations of interest along the PIL are marked by the numbers $1-4$. 


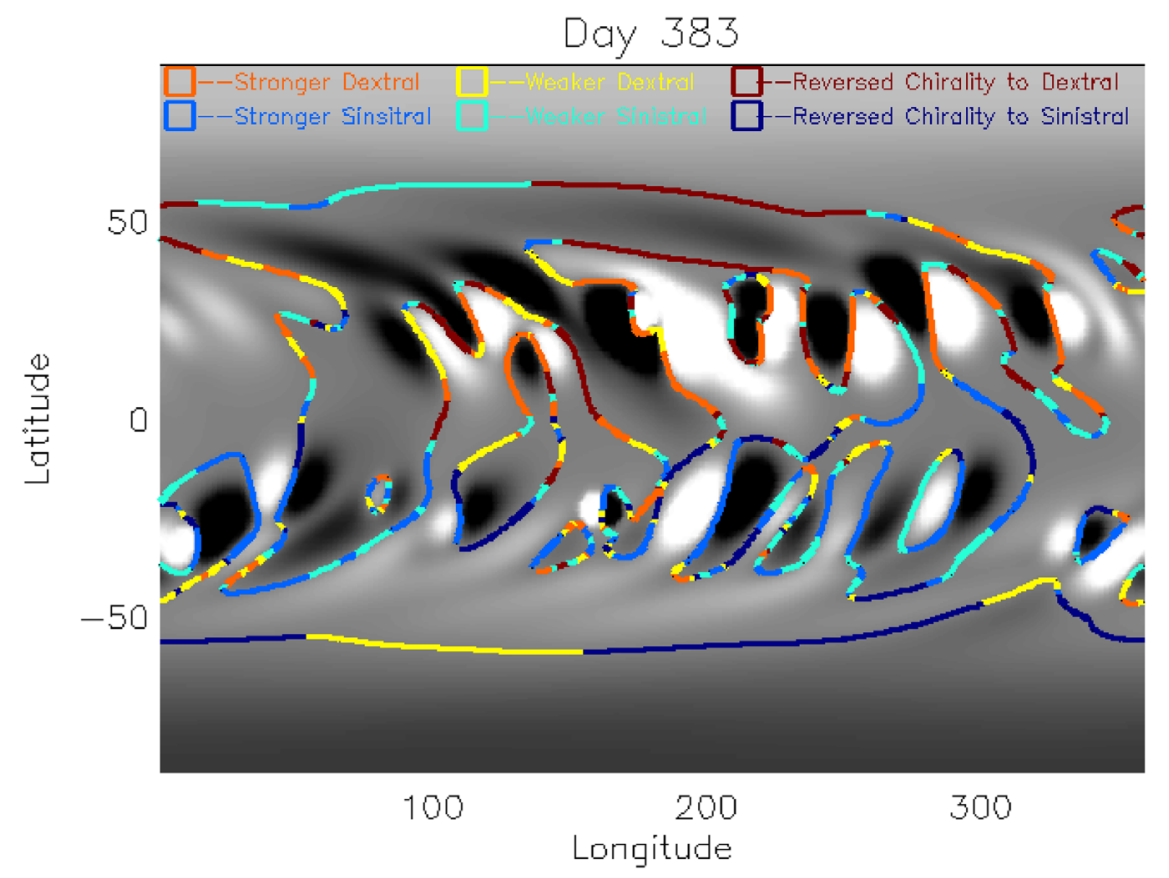

Figure 7. Chirality differences in the field along the PILs for the rising-phase simulations with $\omega_{l}=0 \mathrm{~s}^{-1}$ and $\omega_{l}=5 \times 10^{-6} \mathrm{~s}^{-1}$ on Day 383 . White $/$ black represents positive/negative flux with the values saturated at $\pm 20 \mathrm{G}$. Colored lines denote the path of the PIL where the relative change in skew is given. Orange denotes stronger dextral skew, yellow weaker dextral skew, blue stronger sinistral skew, and cyan weaker sinistral skew; dark red denotes chirality reversals from sinistral to dextral, and dark blue from dextral to sinistral.

On Day 8 (Figures 8(a) and (e)), the field lines that pass over the PIL exhibit weak skew in both simulations, due to the initial potential field configuration. At the locations labeled 1, 2 , and 4 , the field lines mostly connect north-south over the PIL. In contrast, at location 3, near the cusp of the switchback of the PIL, the field lines alter their orientation to connect mainly east-west.

Over the following 40 days with no helicity condensation included (left column), differential rotation acts on the field lines at locations 1,2 , and 4 to produce dextral skew along the initially north-south oriented field lines. Where the field lines initially connect east-west at location 3 , differential rotation has little or no effect on the individual field lines. Instead, it rotates the PIL in a clockwise direction. This rotation in combination with the effect of surface diffusion allows sinistral skew (Figure 6(c)) to develop. The length of this sinistral skew is much less than that of dextral skew, so along the illustrated PIL the observed hemispheric pattern is not reproduced. By Days 38 and 48, these processes lead to strong axial field and predominantly dextral skew along the entire length of the PIL.

Comparing the left and right columns in Figure 8 shows that the SAHC model has two effects. First, at locations 1 and 4, where the field lines initially lie north-south, the injection of positive helicity counters the effect of differential rotation. The field lines throughout the 40-day period mainly lie normal to the PIL or have very weak dextral skew. The most significant difference occurs close to the first cusp in the PIL. When helicity condensation is neglected, the field lines acquire dextral skew at location 2 and sinistral skew at location 3 . When it is included, by Day 28 (Figure 8(f)) the skew at location 2 reverses to be sinistral, while the sinistral skew at location 3 is enhanced. This pattern continues through Days 38 and 48 , with the exact levels of shear changing as flux ropes form and are ejected. At location 3 near the cusp on Day 38, the sinistral skew is slightly weaker for the case $\omega_{l}=5 \times 10^{-6} \mathrm{~s}^{-1}$ than for $\omega_{l}=0 \mathrm{~s}^{-1}$. This occurs because the extra helicity injection forms the flux rope much faster and it is subsequently ejected, removing a large amount of the helicity. Similar differences in the evolution of the field are found throughout the simulations; this is just one example.

\subsection{Current and Magnetic Helicities}

Thus far, we have discussed the chirality produced in the simulations in terms of the skew of coronal field lines, comparing this with the observed hemispheric pattern of filaments. Another observational quantity that can be deduced from vector magnetogram data is the current helicity $(\alpha)$ at the photosphere,

$$
\alpha=\frac{\boldsymbol{j} \cdot \boldsymbol{B}}{B^{2}} .
$$

We currently cannot compare our simulation results with those from observations because the vector-field measurements needed to evaluate $\alpha$ can be made only in strong-field regions. Nevertheless, it is useful to examine the distribution and evolution of the current helicity shown in Figure 9.

Figures 9(a) and (c) show the spatial distribution of current helicity on Day 383 for the cases (a) $\omega_{l}=0 \mathrm{~s}^{-1}$ and (c) $\omega_{l}=$ $5 \times 10^{-6} \mathrm{~s}^{-1}$. Red/blue represents negative/positive values saturated at $\pm 2 \times 10^{-8} \mathrm{~m}^{-1}$. The black lines denote the paths of the PILs at the photosphere. The results in Figures 9(a) and (c) can be compared directly to the skew that is obtained for the field lines passing over the PILs (Figures 6(d) and (h)). From Figure 9(a) it is clear that significant regions of both positive and negative current helicity exist at all latitudes. In the northern hemisphere, negative/positive helicity dominates at low/high latitudes; this latitudinal pattern reverses in the southern hemisphere. In Figure 9(c), with helicity condensation included, less intermingling occurs and negative helicity dominates through most 


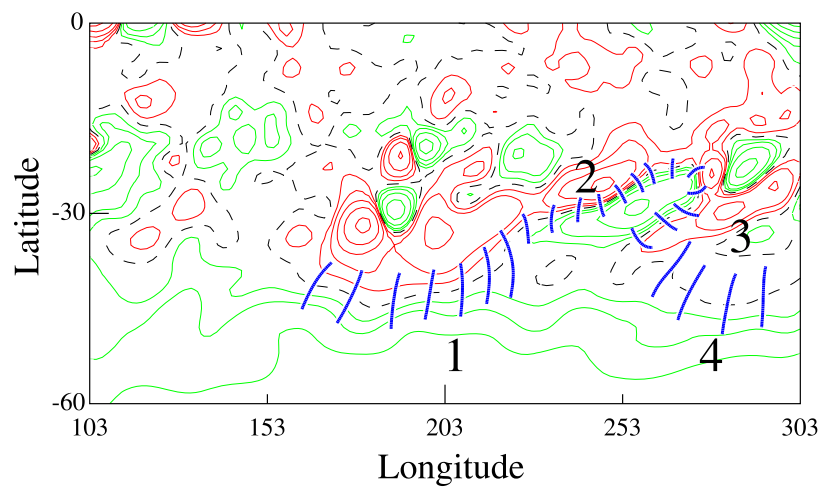

(a)

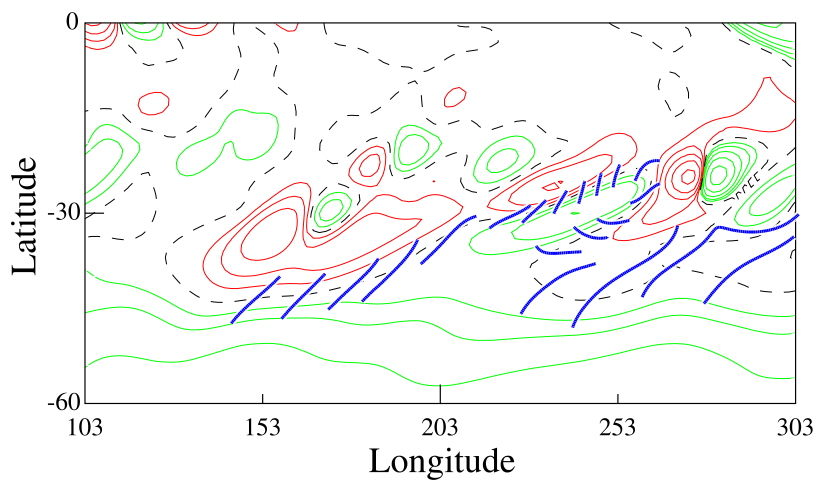

(b)

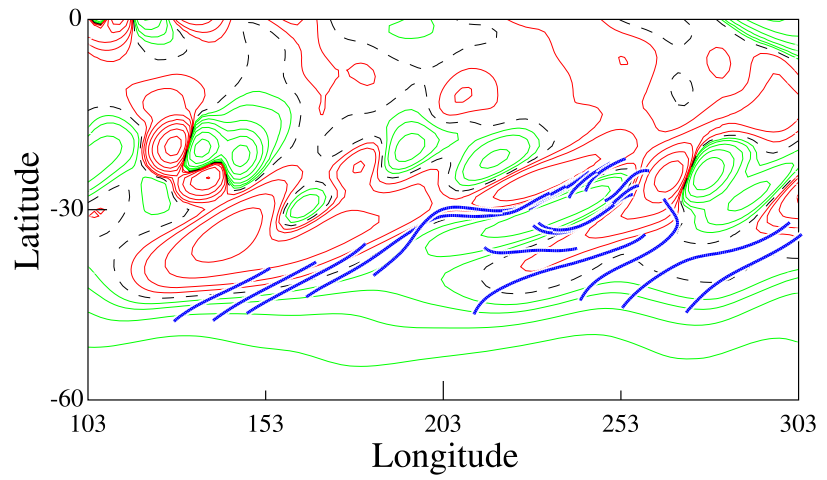

(c)

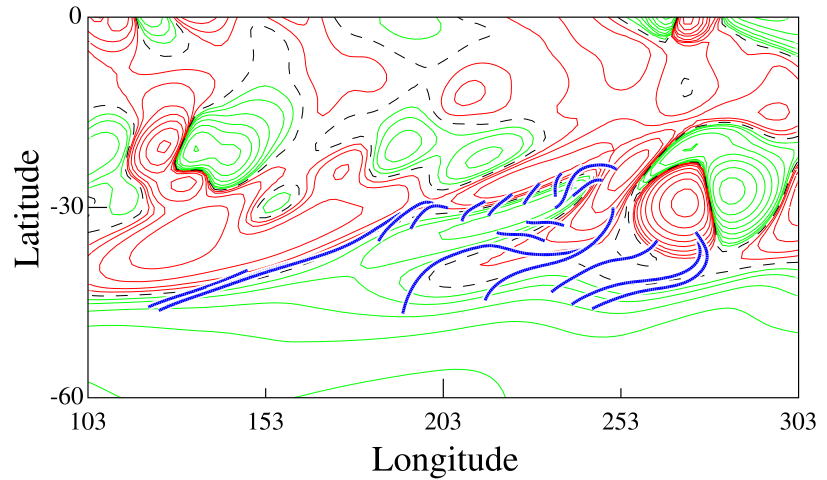

(d)

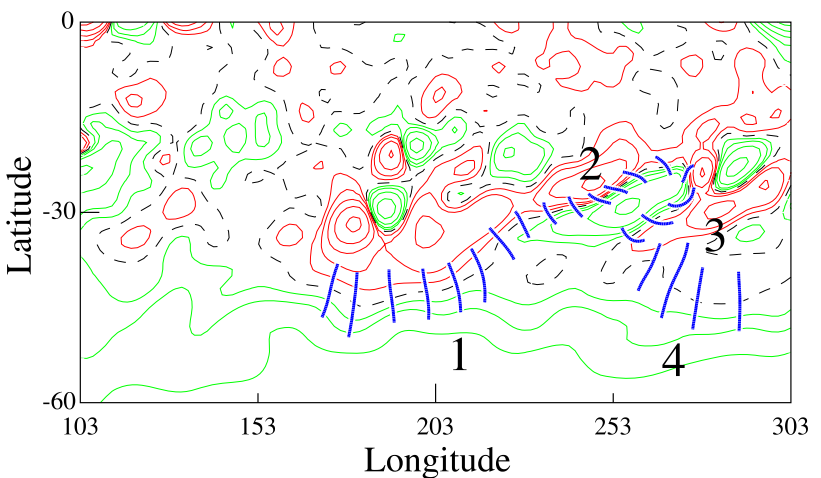

(e)

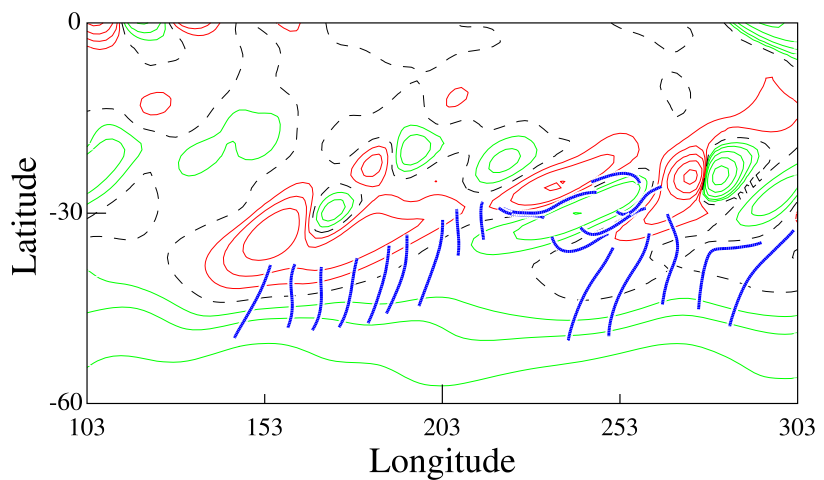

(f)

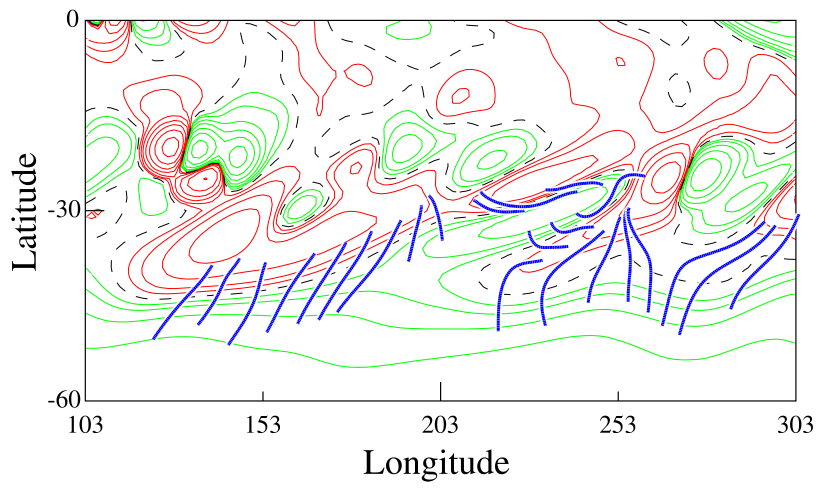

(g)

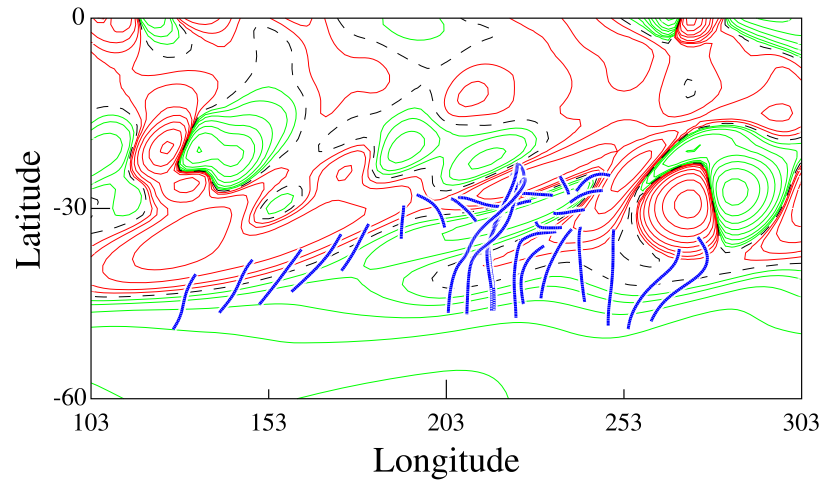

(h)

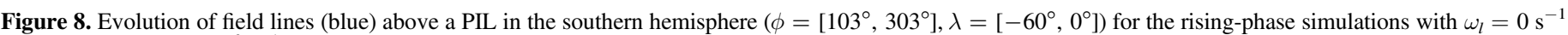

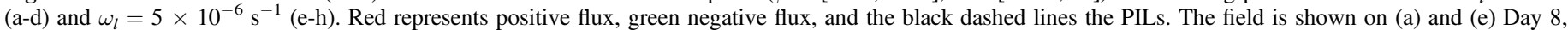
(b) and (f) Day 28, (c) and (g) Day 38, and (d) and (h) Day 48 of the simulation. Key locations that are discussed in the text are labeled 1-4. 


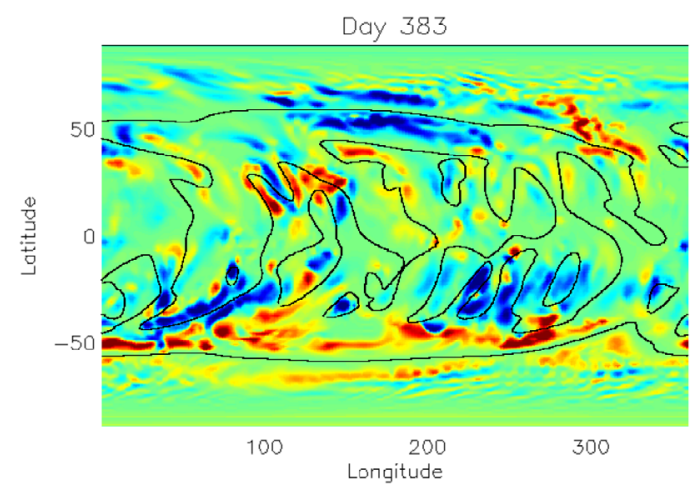

(a) $\omega_{l}=0 \mathrm{~s}^{-1}$

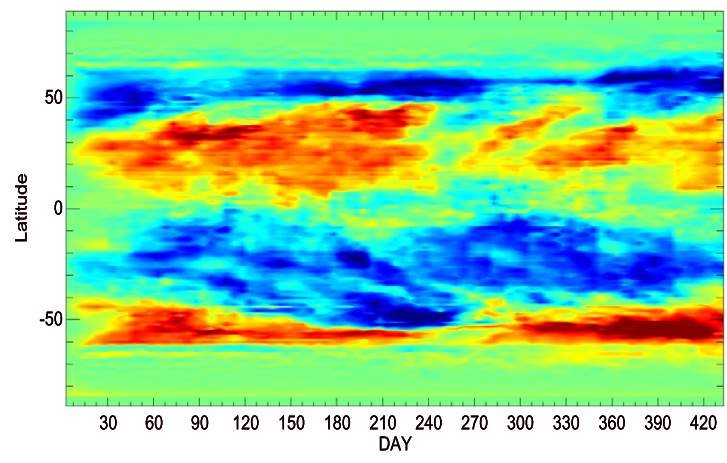

(b) $\quad \omega_{l}=0 \mathrm{~s}^{-1}$

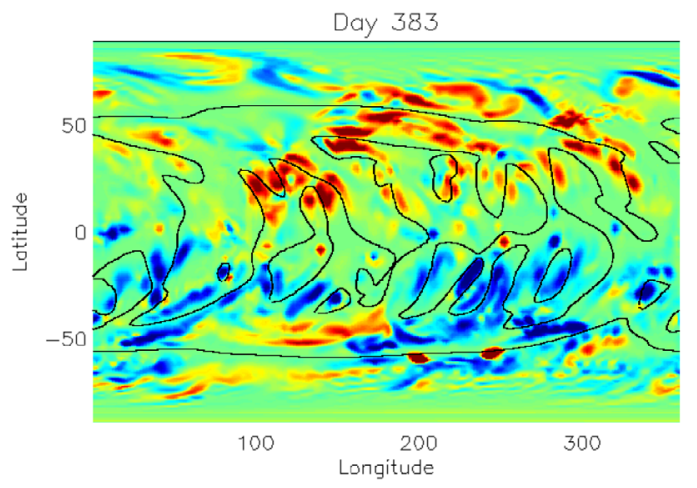

(c)

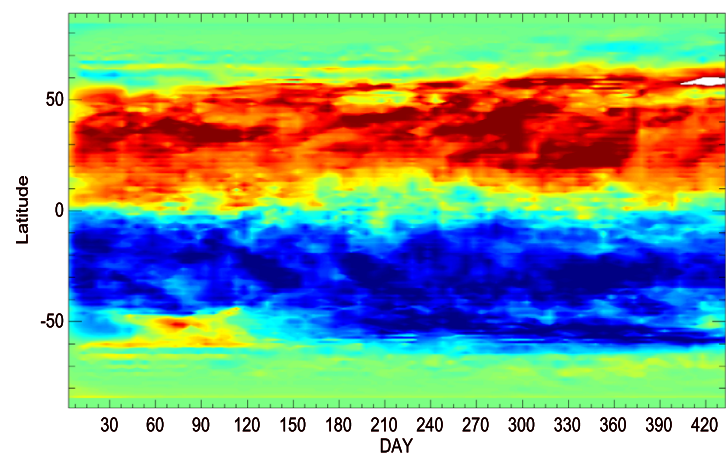

(d)

$$
\omega_{l}=5 \times 10^{-6} \mathrm{~s}^{-1}
$$

Figure 9. Distribution of the current helicity $\alpha$ at the photosphere on Day 383 for the simulations with (a) $\omega_{l}=0 \mathrm{~s}^{-1}$ and (c) $\omega_{l}=5 \times 10^{-6} \mathrm{~s}^{-1}$. A longitudinal average of the current helicity through the entire duration of the simulations is shown for (b) $\omega_{l}=0 \mathrm{~s}^{-1}$ and (d) $\omega_{l}=5 \times 10^{-6} \mathrm{~s}^{-1}$, where the horizontal axis is time in days. Positive/negative values are given by blue/red as shown by the color bar at the right, where the values saturated at $\pm 2 \times 10^{-8} \mathrm{~m}^{-1}$.

of the northern hemisphere, and positive throughout the south. This dominance is not exclusive, however; regions of the opposite sign of current helicity remain in each hemisphere.

A clearer demonstration of the dominant latitudinal profile of current helicity is shown in Figures 9(b) and (d), where the longitudinally averaged current helicity is shown. The vertical axis denotes latitude and the horizontal axis time in days. The colors and saturation levels are identical to those used in Figures 9(a) and (c). The antisymmetry in the current helicity between the northern and southern hemispheres is very apparent in the longitudinal averages. For $\omega_{l}=0 \mathrm{~s}^{-1}$ (Figure 9(b)), the dominant sign of current helicity switches to positive/negative in the northern/southern hemispheres at about $\pm 50^{\circ}$ latitude. This is approximately the latitude where the gradient in differential rotation peaks. At higher latitudes, a band of positive current helicity (sinistral chirality) is produced in the north, and vice versa in the south. In contrast, for $\omega_{l}=5 \times 10^{-6} \mathrm{~s}^{-1}$ (Figure 9(d)), the SAHC mechanism is now able to counter almost completely that of differential rotation. Only small, weak pockets of positive/negative helicity are found in the northern/southern hemisphere.

Figure 10 shows the relative magnetic helicity for three of our simulations as calculated from the rigorous, gaugeindependent formula of Finn \& Antonsen (1985),

$$
H_{r}=\int_{V}\left(\boldsymbol{A}+\boldsymbol{A}_{p}\right) \cdot\left(\boldsymbol{B}-\boldsymbol{B}_{p}\right) d \tau
$$

where $\boldsymbol{A}_{p}$ and $\boldsymbol{B}_{p}=\nabla \times \boldsymbol{A}_{p}$ represent the reference potential field that possesses the same normal magnetic field components on all boundaries as the actual field. Equation (10) is the most

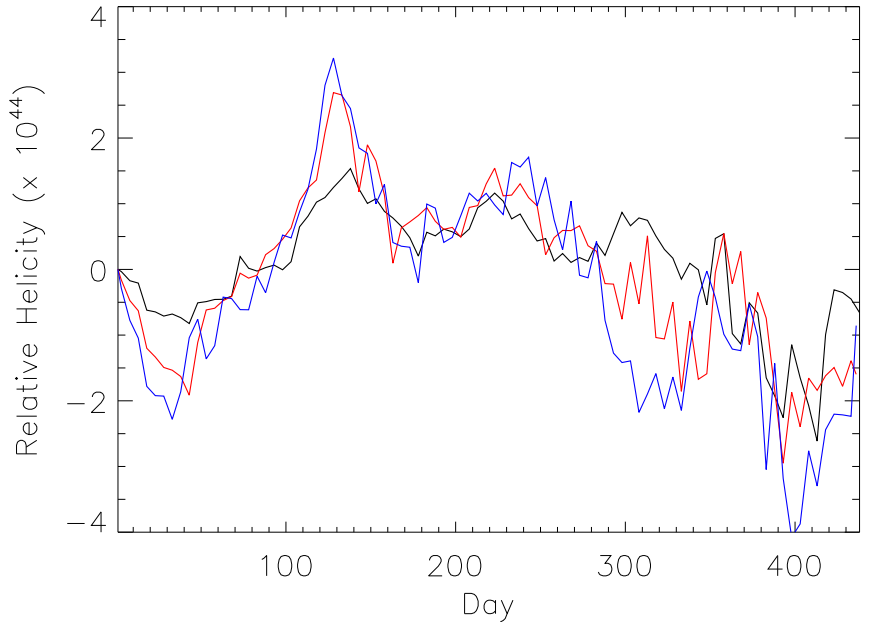

Figure 10. Relative magnetic helicity for the rising-phase simulations. Results are shown for $\omega_{l}=0 \mathrm{~s}^{-1}$ (black), $\omega_{l}=2.5 \times 10^{-6} \mathrm{~s}^{-1}$ (red), and $\omega_{l}=5 \times 10^{-6} \mathrm{~s}^{-1}$ (blue).

convenient expression for computing the relative helicity and its temporal evolution in the $3 \mathrm{D}$ simulation volume, as it is invariant under any gauge transformation of $\boldsymbol{A}$ and $\boldsymbol{A}_{p}$. Since $B_{\theta}=0$ at the latitudinal boundaries in the simulation and we are modeling a full sphere in the longitudinal direction, matching the normal magnetic field component of the potential field to that of the simulation reduces to matching the radial magnetic field at the inner and outer radial boundaries. To obtain the reference potential field that satisfies the correct 
boundary conditions, a relaxation process is carried out here using the Weyl gauge as in the full simulation, until the maximum value of $|\boldsymbol{j}|$ and the volume integral of $|\boldsymbol{j}|$ are less than those found for the initial potential field (which arise due to discretization errors). Note that the integration of Equation (10) is over the whole simulation domain, the volume between the solar surface and the outer boundary, so it includes both open and closed flux. While Equation (10) may be used to quantify the temporal evolution of relative helicity within the simulations, it cannot be applied directly to observations, as in general the coronal magnetic field cannot be measured. To determine the temporal evolution of relative helicity from observations, a helicity flux at the photosphere into the corona must be calculated. A significant complication of this process is that the helicity flux as usually expressed (Berger \& Field 1984) is not gauge invariant. For a comprehensive discussion of this issue, we refer to Pariat et al. (2015).

In Figure 10 the gauge-independent relative helicity from the simulations is shown for $\omega_{l}=0 \mathrm{~s}^{-1}$ (black line), $\omega_{l}=2.5 \times$ $10^{-6} \mathrm{~s}^{-1}$ (red), and $\omega_{l}=5 \times 10^{-6} \mathrm{~s}^{-1}$ (blue). Results for $\omega_{l}=$ $1 \times 10^{-6} \mathrm{~s}^{-1}$ and $\omega_{l}=10 \times 10^{-6} \mathrm{~s}^{-1}$ show similar trends but are not plotted for clarity. The relative helicity in each of the simulations follows a similar trend. The simulation with $\omega_{l}=$ $0 \mathrm{~s}^{-1}$ tends to have the lowest values of helicity, while that with $\omega_{l}=5 \times 10^{-6} \mathrm{~s}^{-1}$ has the highest. Typical values of the relative helicity are on the order of $1 \times 10^{44} \mathrm{Mx}^{2}$ and vary between positive and negative values. Further calculations, in which the modulus of the integrand in Equation (10) is taken, produce a result 10-20 times higher. Since roughly equal amounts of oppositely signed helicity exist in each hemisphere, the low values found in Figure 10 represent the imbalance of helicity between the two hemispheres where both hemispheres are highly nonpotential. This imbalance is due to the wide variety of helicity injection and loss mechanisms acting at any given time. In addition to the calculation shown in Figure 10 using the Weyl gauge, a calculation of relative magnetic helicity has been carried out where the reference potential field in Equation (10) was calculated using the Coulomb gauge (see Pariat et al. 2015, for a discussion on the consequences of using different gauges), but this time not matching the normal field component at the outer boundary. We emphasize that by not matching the outer boundary, this process is not strictly correct. However, results very close to those shown in Figure 10 were found. There was no systematic offset between the magnetic helicities using the Weyl gauge (black line in Figure 10) and that of the Coulomb gauge (with incorrect outer boundary), and the mean absolute difference between them was $4 \%$. This illustrates both the small impact of the outer radial boundary values used in the calculation and the robustness of the gaugeinvariant Finn-Antonsen form of the helicity.

\section{Declining Phase}

Results are now presented for the declining-phase simulations, again considering five separate cases. When included, helicity condensation as described by the SAHC model is applied with the same vorticities and latitudinal sign dependence as assumed for the rising-phase. Graphs showing the variation of the global quantities for the declining phase are not shown as they exhibit the same behavior as we discussed in Section 5.1. The only significant difference is that now the overall level of magnetic flux shows a decreasing trend, with similar variations in the other quantities.

\subsection{Latitudinal Distributions}

Figure 11 shows the distributions of skew versus latitude for the declining-phase simulations, using the same color scale as Figure 5. Results are again shown for (a) $\omega_{l}=0 \mathrm{~s}^{-1}$, (b) $\omega_{l}=2.5 \times 10^{-6} \mathrm{~s}^{-1}, \quad$ (c) $\omega_{l}=5 \times 10^{-6} \mathrm{~s}^{-1}$, and (d) $\omega_{l}=10 \times 10^{-6} \mathrm{~s}^{-1}$. One important difference between the rising- and declining-phase simulations is that the results for the latter now span a wider range of latitudes (up to $\pm 83^{\circ} .1$ ). When only surface flux transport and magnetic flux emergence act on the coronal field (Figure 11(a)), nearly equal amounts of dextral and sinistral skew are produced in each hemisphere. A bimodal distribution forms that is clearly inconsistent with the observed hemispheric pattern of filaments.

When helicity condensation is included at a rate of $\omega_{l}=2.5 \times 10^{-6} \mathrm{~s}^{-1}$ (Figure 11(b)), a latitudinal distribution more consistent with the observed hemispheric pattern occurs. Below $\pm 35^{\circ}$ latitude, dextral/sinistral chirality dominates in the northern/southern hemisphere. The pattern at high latitudes is, however, still inconsistent with the observed hemispheric pattern, as sinistral/dextral chirality dominates in the northern and southern hemispheres. Correspondingly, a bimodal distribution is produced. Increasing the vorticity further, to $5 \times$ $10^{-6} \mathrm{~s}^{-1}$, produces a chirality pattern consistent with observations in the northern hemisphere, with a single peak occurring at low to mid latitudes. Interestingly, this pattern is not mirrored in the southern hemisphere, where a bimodal pattern remains and dextral skew dominates at $-60^{\circ}$ latitude. Only for $\omega_{l}=10 \times 10^{-6} \mathrm{~s}^{-1}$ does a unimodal distribution with dominant dextral/sinistral skew across all latitudes occur in both the northern and southern hemispheres.

Table 2 shows the relative lengths of the PILs that exhibit skew in each hemisphere for the declining-phase simulation. Upon comparing these results with Table 1, it is clear that in the declining phase, for each vorticity a higher amount of minority chirality is produced in each hemisphere. As found for the rising phase, the skewed portions of the PIL satisfy the observed ratio when a vorticity of $2.5 \times 10^{-6} \mathrm{~s}^{-1}$ is applied; however, a much higher vorticity of $10 \times 10^{-6} \mathrm{~s}^{-1}$ is required to obtain the unimodal chirality distribution across all latitudes.

\subsection{PIL Skew Distributions}

To investigate why higher rates of vorticity are required to produce a unimodal pattern of skew in the declining phase, the evolution of the skew along individual PILs is shown in Figure 12. Results are once again shown for the early part of the simulations, on Days 26 (a) and (e) and 166 (b) and (f), and the later stages of the simulations, on Days 301 (c) and (g) and 406 (d) and (h). The paths of the PILs at the level of the photosphere are color-coded according to the chirality of skew (calculated at a height of 10,000 km): red denotes dextral skew, blue sinistral skew, and green weak skew. Panels (a)-(d) show the results for $\omega_{l}=0 \mathrm{~s}^{-1}$, while panels (e)-(h) show results for $\omega_{l}=5 \times 10^{-6} \mathrm{~s}^{-1}$.

On comparing the paths of PILs in Figures 12 ((a) and (e)) with Figures 6 ((a) and (e)), it is apparent that in the declining phase, the PILs initially cover a much wider range of latitudes. As the declining-phase simulation progresses, there is then a general progression for the PILs to be located at lower latitudes 

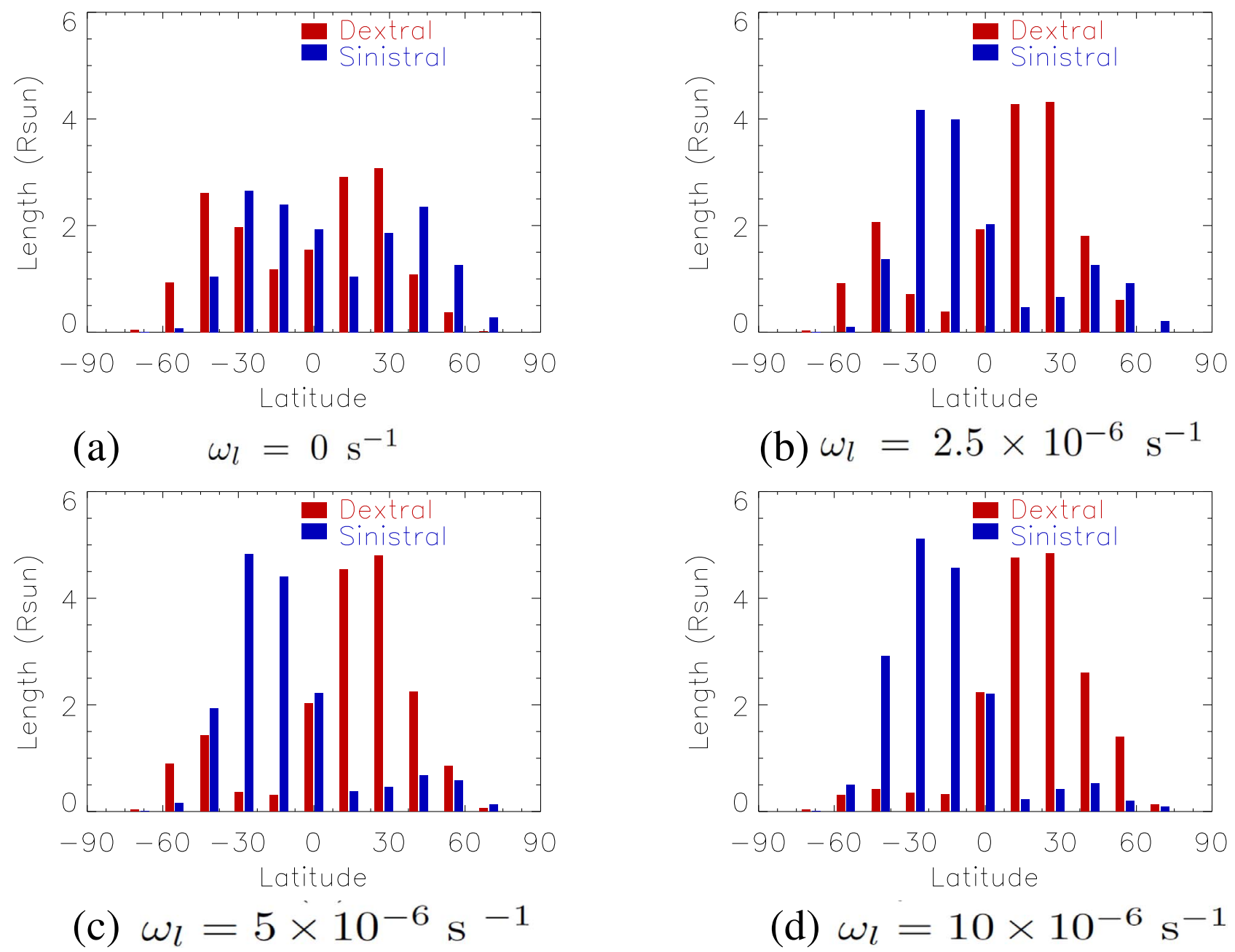

Figure 11. Same as Figure 5, but for the declining-phase simulations.

Table 2

Same as Table 1, but for the Declining-phase Simulations

\begin{tabular}{lcc}
\hline \hline$\omega_{l}$ & $\begin{array}{c}\text { Northern } \\
\text { Dextral/Sinistral }\end{array}$ & $\begin{array}{c}\text { Southern } \\
\text { Dextral/Sinistral } \\
\%\end{array}$ \\
$\mathrm{~s}^{-1}$ & $53 / 47$ & $52 / 48$ \\
\hline 0 & $62 / 38$ & $42 / 58$ \\
$1 \times 10^{-6}$ & $76 / 24$ & $30 / 70$ \\
$2.5 \times 10^{-6}$ & $85 / 15$ & $21 / 79$ \\
$5 \times 10^{-6}$ & $90 / 10$ & $10 / 90$ \\
$10 \times 10^{-6}$ & & \\
\hline
\end{tabular}

due to the strengthening of the polar fields. Upon comparing the northern and southern hemispheres in the declining-phase simulation, a slightly different behavior for the orientation of the PILs is found. In the northern hemisphere between Days 301 and 406, the highest latitude PILs have progressed to reside below $50^{\circ}$, with only small portions lying east-west. In contrast, for the southern hemisphere from Day 116 forward, there is a persistent east-west oriented PIL that nearly extends all the way around the Sun on Day 301. As more trailing polarity flux is pushed poleward in the southern hemisphere, this PIL breaks up and is reduced to extend approximately $120^{\circ}$ in longitude by Day 406. Despite the reduction in length, it remains a persistent feature that sits on or near the peak in the differential rotation gradient.

For $\omega_{l}=0 \mathrm{~s}^{-1}$ (left column), the skew is consistent with that already discussed in Section 5.3: along the north-south oriented PILs, dextral/sinistral skew is found in the northern/southern hemisphere. The opposite skew is found at mid to high latitudes, where along the east-west oriented PILs sinistral/dextral skew is produced in the northern/southern hemisphere. While this is the general rule, exceptions to this occur. The origin of this pattern, due to the sign of magnetic helicity injected along different orientations of the PIL by differential rotation, has already been discussed in Section 5.3.

When helicity condensation is included with a vorticity of $\omega_{l}=5 \times 10^{-6} \mathrm{~s}^{-1}$ (Figures 12(e)-(h)), results similar to those discussed in Section 5.3 are found throughout most of the northern and southern hemispheres. At low to mid latitudes in the northern/southern hemisphere, dextral/sinistral skew is generally much more dominant. In the northern hemisphere at high latitudes, along the east-west oriented PILs, the SAHC mechanism has countered the helicity injected by differential rotation. A notable exception occurs in the southern hemisphere around $-50^{\circ}$ latitude, where throughout the simulation (especially in the later stages) a persistent band of dextral skew exists. This band of dextral skew results in the bimodal distribution for the southern hemisphere that is 


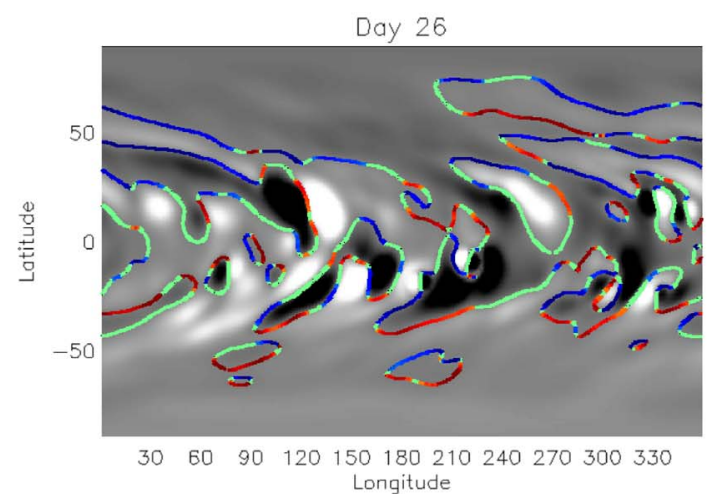

(a)

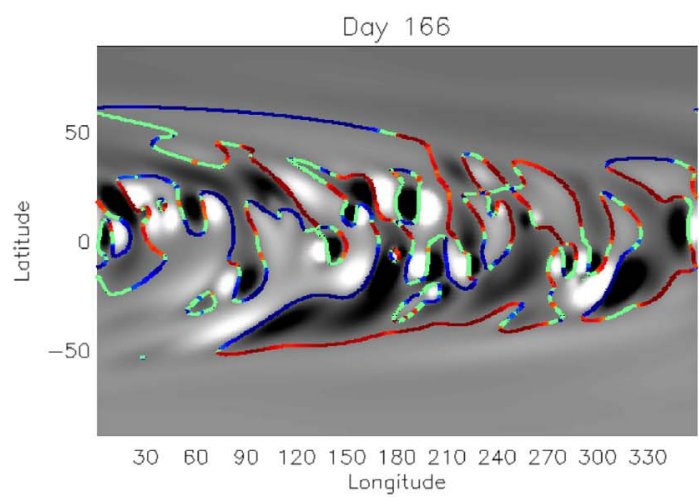

(b)

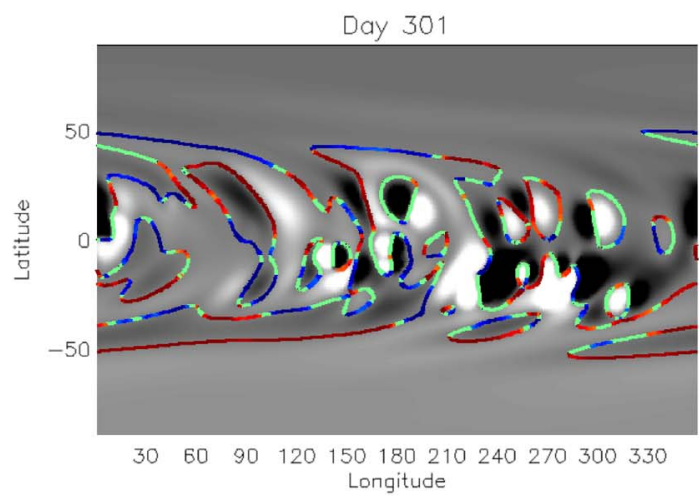

(c)

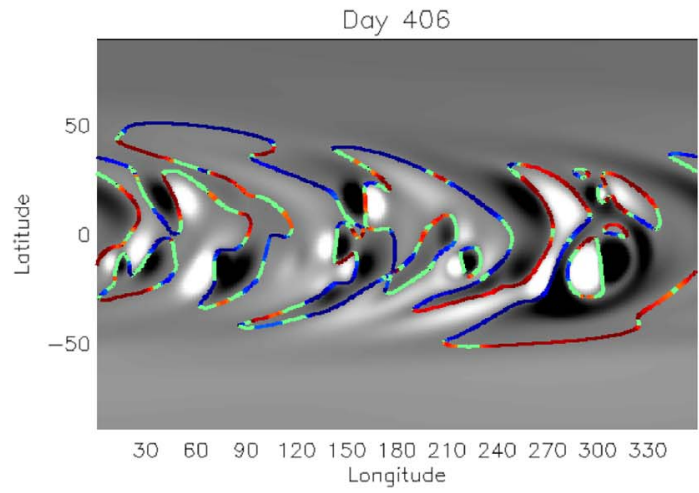

(d)

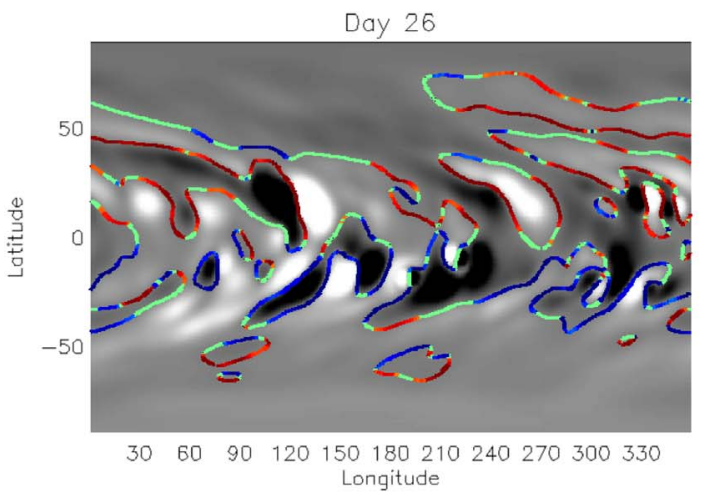

(e)



(f)

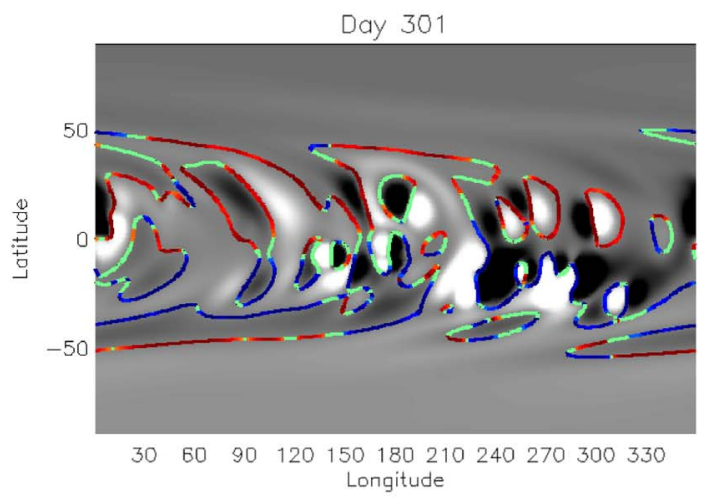

(g)

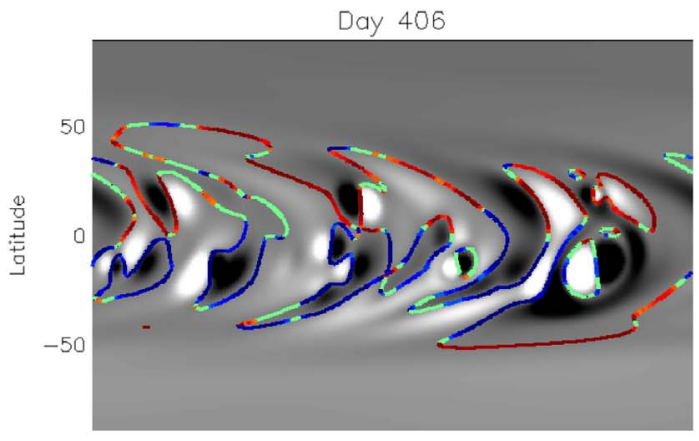

$30 \quad 60 \quad 90 \quad 120150180210240270300330$

(h)

Figure 12. Same as Figure 6, but for the declining-phase simulations, with snapshots displayed on Days 26, 166, 301, and 406.

seen in Figure 11(c). As this PIL consistently lies on or around the peak in the gradient of differential rotation, it is difficult for helicity condensation to overcome it when nonlocal effects due to surface diffusion are also present. However, by increasing the vorticity by just a further factor of two, to $\omega_{l}=10 \times 10^{-6} \mathrm{~s}^{-1}$, differential rotation can be overcome and a dominant sinistral skew is produced at this location (Figure 11(d)). 


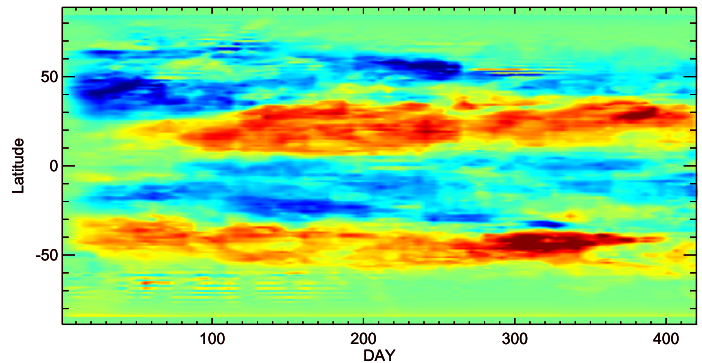

(a) $\omega_{l}=0 \mathrm{~s}^{-1}$

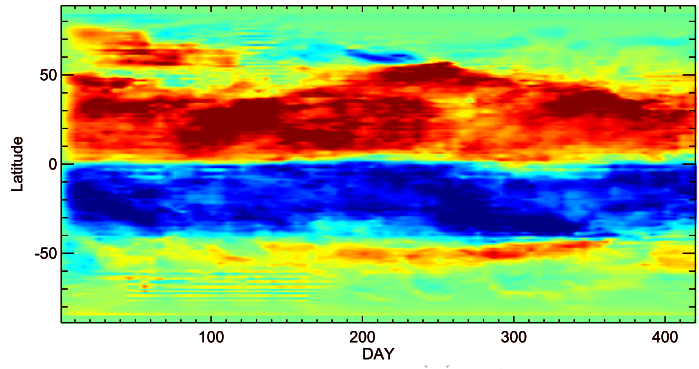

(b) $\omega_{l}=5 \times 10^{-6} \mathrm{~s}^{-1}$

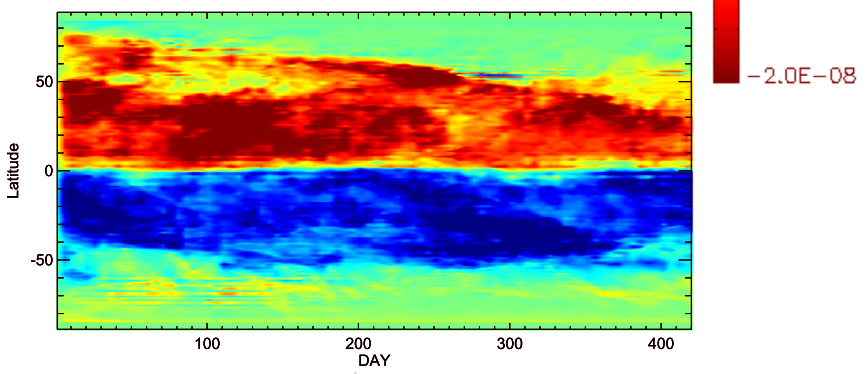

(c) $\omega_{l}=10 \times 10^{-6} \mathrm{~s}^{-1}$

Figure 13. Longitudinal average of the current helicity for the declining-phase simulation for (a) $\omega_{l}=0 \mathrm{~s}^{-1}$, (b) $\omega_{l}=5 \times 10^{-6} \mathrm{~s}^{-1}$, and (c) $\omega_{l}=10 \times$ $10^{-6} \mathrm{~s}^{-1}$, where the horizontal axis is time in days. Positive/negative values are given by blue/red as described by the color panel on the right-hand side, where the values saturated at $\pm 2 \times 10^{-8} \mathrm{~m}^{-1}$.

\subsection{Latitudinal Distribution of Current Helicity}

The latitudinal profile of current helicity in the decliningphase simulations is shown in Figure 13, where the longitudinally averaged current helicity is given for (a) $\omega_{l}=0 \mathrm{~s}^{-1}$, (b) $\omega_{l}=5 \times 10^{-6} \mathrm{~s}^{-1}$, and (c) $\omega_{l}=10 \times 10^{-6} \mathrm{~s}^{-1}$. As used previously, red/blue represents negative/positive values, where the saturation value is set at $\pm 2 \times 10^{-8} \mathrm{~m}^{-1}$. As found for the rising-phase simulations, there is an antisymmetry in the current helicity between the northern and southern hemispheres.

For $\omega_{l}=0 \mathrm{~s}^{-1}$ (Figure 13(a)), the dominant sign of current helicity switches to be positive/negative in the northern/ southern hemispheres around $\pm 35^{\circ}$ latitude. At latitudes above this, positive helicity dominates in the north and negative in the south. This change in sign of helicity occurs at a significantly lower latitude than in the corresponding rising-phase simulation (Figure 9(c)). Upon directly comparing Figure 13(a) with Figure 9(c), it is apparent that in the declining phase there is a stronger preference for the production of positive/negative helicity in the northern/southern hemisphere. This can be explained in terms of the reversal in sign of the polar fields and the changing paths of PILs, such that more PILs have an east-west orientation along the lead arms of switchbacks (see Figure 11 of Mackay et al. 2014). When $\omega_{l}=5 \times 10^{-6} \mathrm{~s}^{-1}$ (Figure 13(b)), the SAHC mechanism can counter almost completely the helicity injected by differential rotation in the northern hemisphere. However, in the southern hemisphere, a strong band of negative helicity persists at approximately $-50^{\circ}$ latitude between days 100 and 350 . This band represents the dextral chirality that lies above a persistent high-latitude east-west PIL in the right column of Figure 12.

Finally, in Figure $13(\mathrm{c})$ the case $\omega_{l}=10 \times 10^{-6} \mathrm{~s}^{-1}$ is shown. With the increased value of vorticity, the SAHC mechanism is able to completely counter that of differential rotation. Only small and weak pockets of positive/negative helicity are found in the northern/southern hemisphere. These results indicate that in the declining phase there is an enhanced likelihood for the production of positive/negative helicity or correspondingly sinistral/dextral skew in the northern/southern hemisphere compared to the rising phase. This can also be seen upon comparing the results in Tables 1 and 2. Therefore this gives increased support to the suggestion of Pevtsov et al. (2003) that in the declining phase the hemispheric pattern is not as strong.

\section{Summary}

To evaluate more globally the results presented in Sections 5 and 6 , a qualitative comparison is now made with observations of the hemispheric pattern of filaments given in Section 2. Overall, observational studies of filament chirality indicate that the hemispheric pattern consists of an approximate 75\%:25\% dependence of dominant to minority chirality in each hemisphere. Such relative values are found for both the rising- and declining-phase simulations in Tables 1 and 2 when a vorticity of $2.5 \times 10^{-6} \mathrm{~s}^{-1}$ is applied. While obtaining the overall average is one measure, an arguably better comparison is the latitudinal distribution of sinistral and dextral chirality.

The results in Figure 1 can be compared to those in Figures 6 and 11 , but care must be taken as fundamentally different quantities are being considered. For the observations, it is the number of filaments exhibiting chirality, while for the simulations, it is the physical length of the PIL. It should also be noted that the simulations present results up to $\pm 69^{\circ} .2$ latitude, while the observations only extend to $\pm 50^{\circ}$ latitude. This is mostly due to the difficulty in determining the observed chirality of filaments from barbs at high latitudes. Taking these differences into account, it is clear that when the vorticity is $2.5 \times 10^{-6} \mathrm{~s}^{-1}$, the simulated distributions for both the rising and declining phase do not match those observed (even though the relative percentage is in good agreement). The simulations produce a bimodal pattern in each hemisphere. For the risingphase simulation, the correct single-peak profile, peaking at $\pm 30^{\circ}$ latitude, is obtained for $\omega_{l}=5 \times 10^{-6} \mathrm{~s}^{-1}$. Using this rate of vorticity also produces a single-peaked profile in the northern hemisphere for the declining-phase simulation, but the higher value of $\omega_{l}=10 \times 10^{-6} \mathrm{~s}^{-1}$ is required for the southern hemisphere. This higher value is due to a specific east-west oriented PIL maintaining itself around $\pm 50^{\circ}$ latitude, where the peak gradient in differential rotation occurs. As such, it is a special case particular to the simulated time period. Therefore we may conclude that the correct latitudinal profile with a peak around $\pm 30^{\circ}$ latitude is produced when a vorticity of $\omega_{l}=5 \times 10^{-6} \mathrm{~s}^{-1}$ or higher is applied. In qualitative agreement with Pevtsov et al. (2003), the declining-phase simulation also produces a larger fraction of minority chirality in each hemisphere than in the rising-phase, as can be seen by comparing Tables 1 and 2 . 


\section{Conclusions}

We have investigated through numerical simulations the consequences of helicity condensation for the Sun's hemispheric pattern of filament chirality. Observations show that dextral/sinistral filaments, containing mostly negative/positive helicity, dominate in the northern/southern hemisphere. This pattern is closely related to the injection and transport of magnetic helicity across the solar surface and its localization onto PILs. To investigate the role of helicity condensation, a global nonpotential evolution model of the Sun's large-scale magnetic field has been applied. The model simulates the evolution of the Sun's photospheric and coronal magnetic fields as new magnetic flux emerges on the Sun and is acted upon by differential rotation, meridional circulation, and surface diffusion. In response to these processes that inject energy and helicity into the atmosphere, the coronal magnetic field evolves through a sequence of nonlinear force-free states. In conjunction with these processes, a large-scale, temporally and spatially averaged, statistical approximation to the full helicity-condensation process was applied. We refer to this approach as the statistically averaged helicity-condensation (SAHC) model, to distinguish it from the full helicitycondensation process of Antiochos (2013). As the SAHC model is an approximation, it is not meant to replicate the complex convection and reconnection dynamics that occur over short temporal and spatial scales within the full helicitycondensation model. The SAHC model therefore is defined in terms of two parameters, the average vorticity and average length scale of the convection cells, which are not resolved within our numerical simulations. At the present time such a parameterized model is the only feasible approach to consider the effects of helicity condensation in competition with differential rotation, meridional flow, flux emergence, and surface diffusion over long periods of time. As the length scale of convective cells is well constrained from observations, we only varied the vorticity of the underlying convective cells as a free parameter.

To investigate the role of helicity condensation on the chirality of the global magnetic field, two periods of $16 \mathrm{CRs}$ were simulated, one in the rising phase of the solar cycle, the other in the declining phase. We here neglected the internal helicity of new emerging active region bipoles as we wished to investigate the competing effects of helicity condensation and differential rotation. In the simulations, the characteristic and statistical properties of the chirality and the physical length of the PILs that exhibited either dextral or sinistral skew were then quantified at each latitude.

In both the rising- and declining-phase simulations, when only surface flux transport processes and magnetic flux emergence (without self-helicity) act on the coronal field, the chirality produced in each hemisphere exhibited an antisymmetric bimodal distribution. This distribution is inconsistent with the observed hemispheric pattern. When helicity condensation as described by the SAHC model is included, it produces a coronal field that is significantly more energized. Moreover, in both the rising- and declining-phase simulations, when the vorticity has a magnitude $\omega_{l}=2.5 \times 10^{-6} \mathrm{~s}^{-1}$, $75 \%-80 \%$ of the PIL skew is of dominant chirality in each hemisphere. Although this is the correct percentage, at high latitudes minority chirality dominates, inconsistent with observations, and a bimodal pattern occurs. To produce a chirality distribution that is consistent with observations over all latitudes, the vorticity of the supergranular cells must be increased to at least $5 \times 10^{-6} \mathrm{~s}^{-1}$. The chirality pattern then became antisymmetric across the equator, with a single peak occurring at approximately $\pm 30^{\circ}$ latitude. This qualitatively matches the observed distributions seen in Figure 1. Even with this relatively high value of vorticity, more than five times greater than the peak value in the gradient of differential rotation, pockets of minority chirality remained. Results also indicate that in the declining phase there is an enhanced likelihood for the production of minority chirality skew in each hemisphere compared to the rising phase. This supports observations that suggest that the hemispheric pattern may be weaker in the declining phase (Pevtsov et al. 2003).

The simulations presented here illustrate that the hemispheric pattern of filaments is likely to be the result of a complex interrelationship between a number of competing processes that inject helicity into or remove helicity from the corona. Even though the SAHC model injects only negative/positive helicity into the northern/southern hemisphere, a variety of changes in the field can occur when additional local (differential rotation, flux emergence, and flux-rope eruptions) and nonlocal (surface diffusion) effects are taken into account. One important feature of the SAHC model is that as the vorticity increases through the values $1-5 \times 10^{-6} \mathrm{~s}^{-1}$, the physical lengths of the sheared PILs at high latitudes decrease. In contrast, the fields at low latitudes become more strongly sheared. This occurs because at high latitudes, the helicity-condensation process described by the SAHC model just counters the sign and magnitude of helicity injected by differential rotation along mostly east-west oriented PILs. At low latitudes, the two processes complement one another along mostly north-south oriented PILs.

In this paper, we have carried out the most comprehensive tests to date of the SAHC model in global-scale simulations. Through this, we have determined a lower bound for the rate of small-scale helicity injection by convective flows and/or magnetic-carpet flux emergence that enables helicity condensation to overcome differential rotation and produce the correct latitudinal distribution for the hemispheric pattern of filaments. Future studies should investigate whether, and by how much, this bound can be lowered when the self-helicity of emerging active region bipoles is included. We will consider this in a follow-up study. In addition, due to the wide range of helicityloss and -gain mechanisms that are at work within these simulations, it is important to carry out a study of the helicity budget so that the competing loss and gain mechanisms can be fully quantified. From an observational perspective, future validation of the SAHC model must also consider one-to-one comparisons of the chirality produced along PILs with actual observations of filament chirality. In particular, this could be carried out with the data set developed by Pevtsov et al. (2003). Simulations that directly compare the SAHC model with that of the full helicity-condensation process also need to be carried out, to compare and contrast the two approaches and to verify the approximations applied to derive the SAHC model. We note that when a spatially uniform value of small-scale helicity injection is used in each hemisphere, two separate rates of effective vorticity are required to produce the observed percentage relationship $\left(\omega_{l}=2.5 \times 10^{-6} \mathrm{~s}^{-1}\right)$ and the observed latitudinal distribution $\left(\omega_{l}=5 \times 10^{-6} \mathrm{~s}^{-1}\right)$. This suggests that a spatially varying profile that transitions smoothly through zero at the equator and increases in magnitude with latitude may reproduce both of these observational features. The possible 
form of such a profile will be considered in future studies. Finally, future observational studies should focus on examining the small-scale helicity injection at the solar surface to determine both its magnitude and latitudinal variation relative to the differential-rotation gradient on the Sun.

D.H.M. and A.R.Y. thank the STFC and the Leverhulme Trust for their financial support, while C.R.D. and S.K.A. acknowledge NASA's LWS TR\&T and H-SR programs for supporting their contributions to the work. D.H.M. and C.R.D. participated in a team investigation of solar prominences at the International Space Science Institute, where this collaboration was conceived and initiated.

\section{Appendix A Initial Condition}

To produce the initial photospheric field distribution on the start day of each simulation, three synoptic magnetograms are required, as shown in Figure 2 and illustrated for the risingphase simulation (a similar process is carried out for the declining-phase simulation). In addition to the rotation from which we start (CR 1930), the CRs before and after are required. In Figure 2(a) these rotations are joined together where the direction of time in the map is from right to left. To produce an approximate daily map, a reference Carrington longitude $\left(\phi_{0}\right)$ is specified in CR 1930. This reference Carrington longitude $\phi_{0}$ specifies the start day of the simulation (1997 December 11) when this longitude lay at central meridian on the Sun on the given day. In Figure 2(a) the white vertical dashed line at $180^{\circ}$ denotes the start date of the simulation, while the lines at $0^{\circ}$ and $360^{\circ}$ denote the boundaries between the maps.

To compensate for the time difference between the left and right edges of rotation map CR 1930, all points to the right of $\left(\phi_{0}\right)$ need extra differential rotation applied, while points to the left require differential rotation to be removed. The amount of differential rotation applied/removed depends on the colatitude $(\theta)$. The transformation is given by

$$
\phi=\phi_{0}+\frac{\Omega(\theta)+\Omega_{0}}{\Omega_{o}}\left(\phi_{m}-\phi_{0}\right),
$$

where the original point is located at $\left(\phi_{m}, \theta\right), \Omega(\theta)$ is the differential rotation profile in the Carrington frame, and $\Omega_{0}=13.2 \mathrm{deg} \mathrm{day}^{-1}$ is the rotation rate of the Carrington frame. The transformed coordinate $\phi$ lies at the same latitude as the original point. In applying this transformation, the effects of meridional flow and surface diffusion over the 13-day difference between the left/right edges and the central longitude are neglected. This is reasonable as both meridional flow and surface diffusion act over much longer timescales than differential rotation.

In Figure 2(b) the resulting transformation can be seen: the dash-dotted white lines denote the deformation of the boundaries given in (a). By applying this operation, we produce an approximation of the radial magnetic field on the date given of the reference longitude $\left(\phi_{0}\right)$. The vertical dashed lines in (b) now give the boundary of the corrected radial magnetic field that is cut out and used to construct the initial potential field. Tests by Yeates et al. (2007) have shown that this is sufficiently accurate to reproduce the subsequent evolution of the photospheric field over long periods of time.

\section{Appendix B New Active Region Bipoles}

To determine the properties of the new active regions from one $\mathrm{CR}$ to the next, a semi-automated procedure is applied: (i) Successive synoptic magnetograms are compared to one another, and (ii) new bipolar regions are identified. Once a new region is identified, its flux, day of central meridian passage, longitude and latitude, tilt angle, and half-separation between peaks are determined. This process tends to pick up only large regions. However, previous studies have shown that this is sufficient to reproduce the main features of the large-scale field over long periods of time.

Once identified, the magnetic bipoles are inserted into the simulation, assuming that the peaks in the polarities lie at the same latitude (i.e., the tilt angle is zero), using the functional form

$$
\begin{aligned}
& A_{x}=\beta \exp (0.5) B_{o} z \exp (-2 \epsilon) \\
& A_{y}=\exp (0.5) B_{o} \rho \exp (-\epsilon) \\
& A_{z}=-\exp (0.5) \beta B_{o} x \exp (-2 \epsilon),
\end{aligned}
$$

where

$$
\epsilon=\frac{\left(x^{2}+z^{2}\right) / 2+y^{2}}{\rho^{2}},
$$

and $\rho$ is the half-separation between the two peaks of flux, $\beta$ is a twist parameter that controls the self-helicity of the bipoles, and $B_{o}$ is the peak field strength of the bipole determined from the observed flux. In expressing the bipoles in this form, we have used the simulation coordinate system $x=\phi$, $y=-\ln [\tan (\theta / 2)]$, and $z=\ln \left(r / R_{\odot}\right)$. The addition of tilt to represent Joy's law occurs through a rotation of the above equations.

During the process of insertion, the new bipoles are inserted as isolated flux systems into the preexisting coronal field. In a crude manner, this represents new emerging flux on the Sun pushing away any preexisting flux as it emerges. Once this occurs, the bipole quickly reconnects with the surrounding field. This insertion technique therefore allows us to add new photospheric flux while also maintaining the prior connectivity of the coronal field. The process enables us to carry out long-term simulations that remain representative of what occurred on the Sun without having to reset the coronal field. As a consequence, we can then follow the transport of magnetic energy and helicity across the surface of the Sun over long periods of time.

\section{Appendix C \\ Comparison of the Photospheric Field with Observations}

Figure 3 compares the radial magnetic field distributions produced by the simulation with the original CR synoptic magnetograms. The radial magnetic field from the simulation (left column) represents a snapshot of the simulated global magnetic field at a given instant in time. In contrast, the CR synoptic magnetograms (right column) provide a 27.3-day time representation and do not represent a single instant in time. Time runs from right $\left(360^{\circ}\right.$ longitude $)$ to left $\left(0^{\circ}\right.$ longitude $)$.

Taking into account these differences, the comparison can be made for the rising-phase simulation on (a) Day 3 of the simulation and CR 1930, (b) Day 193 and CR 1937, and finally (c) Day 408 and CR 1945. In each plot the black vertical dashed line denotes the longitude that lies at central meridian 
for both the simulation and CR synoptic magnetograms. White/black represents positive/negative flux with the fields saturated at $\pm 20 \mathrm{G}$.

Due to the difference in the temporal nature of the maps, care must be taken to make a fair comparison. In the synoptic magnetograms, the data at any longitude lying to the right of the vertical dashed line have occurred at an earlier time than that at the same longitude in the simulation. Therefore the simulation will have experienced more differential rotation, meridional flow, and surface diffusion than that seen in the observations. Longitudes farther from the dashed line have had more time passed between the two determinations and will have greater differences between them. Taking this into account when starting at the dashed line and moving to the right, the simulation reproduces the main flux patterns seen in the observations. Note that the CR maps should show more complexity farther from the dashed lines than the simulation. This complexity in the simulation has been smoothed out through the action of the large-scale surface diffusion. This is most apparent for latitudes above $\pm 50^{\circ}$.

In contrast, moving to the left of the dashed line in the synoptic magnetograms represents moving to later times than that represented by the simulation. Therefore the simulation has not yet reached this time and exhibits less differential rotation, meridional flow, and surface diffusion than the observations. There are also more significant differences due to new bipoles that are present in the magnetograms but are not yet present in the simulation. This occurs because new bipoles are emerged on their day of central meridian passage because we lack information as to when they first appeared on the Sun. As the simulation has not yet reached this time, they are yet to be emerged, but will do so during the following days.

While the large-scale field is faithfully represented, more significant differences exist between the simulation and observations when considering small-scale features of the surface field. The differences are most apparent on Days 193 and 408 (Figures 3(b) and (c)), where the small-scale features are absent from the simulation. The small-scale features seen at both low and high latitudes in the observations represent the magnetic network of the Sun. They are present on Day 3 (Figure 3(a)) of the simulation, as at this time the field is close to that prescribed in the initial condition. Insufficient time has passed for the small-scale features to be diffused away by the large-scale surface diffusion term in the flux transport model. Because the flux transport model does not have a mechanism to regenerate the network-scale features, they are absent from the simulation once those in the initial condition have been removed. Some global magnetic field models have incorporated both the maintenance and evolution of the Sun's magnetic network (Schrijver 2001; Meyer \& Mackay 2016). However, for the present study, which aims to consider large-scale longlived features, this is not necessary and so is not included.

\section{ORCID iDs}

Duncan H. Mackay (i) https://orcid.org/0000-0001-6065-8531 C. Richard DeVore (ib https://orcid.org/0000-0002-4668-591X Spiro K. Antiochos (iD https://orcid.org/0000-0003-0176-4312 Anthony R. Yeates (ib https://orcid.org/0000-0002-2728-4053

\section{References}

Antiochos, S. K. 2013, ApJ, 772, 72

Berger, M. A., \& Field, G. B. 1984, JFM, 147, 133

DeVore, C. R. 2000, ApJ, 539, 944

Duvall, T. L., Jr. 1979, SoPh, 63, 3

Duvall, T. L., Jr., \& Gizon, L. 2000, SoPh, 192, 177

Finn, J. M., \& Antonsen, T. M. 1985, CoPPC, 9, 111

Gaizauskas, V. 1998, in ASP Conf. Ser. 150, New Perspectives on Solar Prominences, ed. D. F. Webb, B. Schmieder, \& D. M. Rust (San Francisco, CA: ASP), 257

Gizon, L., \& Duvall, T. L., Jr. 2003, in ESA Special Pub. 517, GONG 2002, Local and Global Helioseismology: The Present and Future, ed. H. Sawaya-Lacoste (Noordwijk: ESA), 43

Knizhnik, K. J., Antiochos, S. K., \& DeVore, C. R. 2015, ApJ, 809, 137 Knizhnik, K. J., Antiochos, S. K., \& DeVore, C. R. 2017a, ApJ, 835, 85 Knizhnik, K. J., Antiochos, S. K., DeVore, C. R., \& Wyper, P. F. 2017b, ApJL, 851, L17

Komm, R., Howe, R., Hill, F., et al. 2007, ApJ, 667, 571

Leighton, R. B. 1964, ApJ, 140, 1547

Leroy, J.-L., Bommier, V., \& Sahal-Brechot, S. 1983, SoPh, 83, 135

Leroy, J.-L., Bommier, V., \& Sahal-Brechot, S. 1984, A\&A, 131, 33

Mackay, D., \& Yeates, A. 2012, LRSP, 9, 6

Mackay, D. H., DeVore, C. R., \& Antiochos, S. K. 2014, ApJ, 784, 164

Mackay, D. H., Gaizauskas, V., \& van Ballegooijen, A. A. 2000, ApJ, 544, 1122

Mackay, D. H., \& van Ballegooijen, A. A. 2001, ApJ, 560, 445

Mackay, D. H., \& van Ballegooijen, A. A. 2005, ApJL, 621, L77

Mackay, D. H., \& van Ballegooijen, A. A. 2006a, ApJ, 641, 577

Mackay, D. H., \& van Ballegooijen, A. A. 2006b, ApJ, 642, 1193

Martin, S. F. 2003, AdSpR, 32, 1883

Martin, S. F., Bilimoria, R., \& Tracadas, P. W. 1994, in Solar Surface Magnetism, ed. R. J. Rutten \& C. J. Schrijver (Dordrecht: Kluwer), 303 Meyer, K. A., \& Mackay, D. H. 2016, ApJ, 830, 160

Pariat, E., Valori, G., Démoulin, P., \& Dalmasse, K. 2015, A\&A, 580, A128 Pevtsov, A. A., Balasubramaniam, K. S., \& Rogers, J. W. 2003, ApJ, 595, 500 Pevtsov, A. A., Canfield, R. C., \& Metcalf, T. R. 1995, ApJL, 440, L109

Rust, D. M. 1967, ApJ, 150, 313

Schrijver, C. J. 2001, ApJ, 547, 475

Schrijver, C. J., Title, A. M., van Ballegooijen, A. A., Hagenaar, H. J., \& Shine, R. A. 1997, ApJ, 487, 424

Sheeley, N. R., Jr. 2005, LRSP, 2, 5

Snodgrass, H. B. 1983, ApJ, 270, 288

van Ballegooijen, A. A., Priest, E. R., \& Mackay, D. H. 2000, ApJ, 539, 983 van Driel-Gesztelyi, L., \& Green, L. M. 2015, LRSP, 12, 1

Yang, W. H., Sturrock, P. A., \& Antiochos, S. K. 1986, ApJ, 309, 383

Yeates, A. R., \& Mackay, D. H. 2009, ApJ, 699, 1024

Yeates, A. R., \& Mackay, D. H. 2012, ApJL, 753, L34

Yeates, A. R., Mackay, D. H., \& van Ballegooijen, A. A. 2007, SoPh, 245, 87

Yeates, A. R., Mackay, D. H., \& van Ballegooijen, A. A. 2008, SoPh, 247, 103

Zhao, L., DeVore, C. R., \& Antiochos, S. K. 2015, ApJ, 805, 61

Zirker, J. B., Martin, S. F., Harvey, K., \& Gaizauskas, V. 1997, SoPh, 175, 27 